September 2, 2018

\title{
Aspects of superconformal field theories in six dimensions
}

\author{
P.J. Heslop \\ Institut für Theoretische Physik der Universität Leipzig
}

\begin{abstract}
We introduce the analytic superspace formalism for six-dimensional $(N, 0)$ superconformal field theories. Concentrating on the $(2,0)$ theory we write down the Ward identities for correlation functions in the theory and show how to solve them. We then consider the four-point function of four energy momentum multiplets in detail, explicitly solving the Ward identities in this case. We expand the four-point function using both Schur polynomials, which lead to a simple formula in terms of a single function of two variables, and (a supersymmetric generalisation of) Jack polynomials, which allow a conformal partial wave expansion. We then perform a complete conformal partial wave analysis of both the free theory four-point function and the AdS dual four-point function. We also discuss certain operators at the threshold of the series a) unitary bound, and prove that some such operators can not develop anomalous dimensions, by finding selection rules for certain three-point functions. For those operators which are not protected, we find representations with which they may combine to become long.
\end{abstract}




\section{Introduction}

Since the discovery of the $A d S / C F T$ correspondence [1-3] relating conformal field theories to supergravity, string theories or $\mathrm{M}$ theories on an $A d S \times S$ background, there has been rapid progress in the investigation of conformal field theories in dimensions larger than two.

The bulk of the analysis on the superconformal field theory side of the corespondence has concentrated on the four dimensional $N=4$ supersymmetric Yang-Mills theory (SYM) and in particular its correlation functions (see [4-6] for reviews). This theory is of great interest for a number of reasons. It has the largest possible amount of flat space supersymmetry in four dimesnions, it is uniquely determined by the coupling constant and the gauge group and it is superconformally invariant even as a quantum theory [7-9]. In particular, it is conjectured to be dual to IIB string theory on $A d S_{5} \times S^{5}$ and so both sides of the AdS/CFT conjecture are at least in principle well-defined theories, allowing the possibility of testing the conjecture.

Less well understood is the conjectured duality between $\mathrm{M}$ theory on $A d S_{7} \times S^{4}$ and a six dimensional superconformal field theory with $(2,0)$ supersymmetry. Neither side of this conjecture is well understood. On the $A d S$ side one knows little about $\mathrm{M}$ theory beyond its low energy limit, supergravity. On the field theory side one knows little beyond the free theory, that of the $(2,0)$ tensor supermultiplet, which was first written down in [10] and reformulated in a suitable harmonic superspace in $[11,12]$. This is believed, however to be the world volume theory of the $\mathrm{M}$ theory 5-brane at low energies. Superconformal symmetry however provides a possible way in to the study of the six dimensional conformal field theory, which can be compared with supergravity results and indeed previous work in this area can be found in [13-16].

Harmonic and analytic superspaces in four space-time dimensions were introduced in [17]. A superfield on analytic superspace which is Grassmann analytic and analytic on the internal variables can also be thought of as an unconstrained superfield (but still analytic in the internal coordinates) on analytic superspace which has a reduced number of odd coordinates. This is similar to the way in which a chiral superfield can be written as an unconstrained superfield on chiral superspace. The general theory of such superspaces realised as coset spaces of complexified superconformal groups was developed in $[18,19]$ (see also [20]).

The analytic superspace formalism is particularly well suited for the study of superconformal symmetry in four dimensions. The advantages of using analytic superspaces are firstly that the full superconformal symmetry is manifest $[18,19,21]$. Secondly, if one takes analytic superspace seriously as a (complex) superspace (rather than simply considering Grassmann analytic superfields on harmonic superspace) one can give all superfields on analytic superspace (even long ones). In general the superfields will transform linearly under finite dimensional irreducible representations of supergroups (so they carry superindices) and remarkably one finds that all irreducible unitary superconformal representations can be given in this way and that furthermore they are all unconstrained (other than than the requirement of analyticity in all the complex variables) $[22,23]$. Furthermore (for even number of supersymmetries $N$ ) there is one analytic superspace which is singled out as a (subset of the) super Grassmanian of subspaces of halfdimension (that is of dimension $(2 \mid N / 2)$ ). These are the natural analogues of Minkowski space for the superconformal case. Indeed the Minkowski space techniques of [24,25] can be adapted 
in the solution of Ward identities.

In a series of papers summarised in [6] many aspects of $N=4$ SYM involving half BPS operators were examined using analytic superspace. Following the results of $[22,23]$ these results were extended to more general operators on analytic superspace [26-28]. A summary of these latter results is given in [29].

Of particular interest in the $N=4$ SYM case has been the four-point function of energymomentum multiplets [30-42]. This is something which can be calculated in the large $\mathrm{N}$ limit using gauged supergravity $[30,33]$ yet it contains within it information about all operators occurring in the OPE of two energy-momentum operators (which for example includes operators dual to string states.) It thus provides an important place to both learn from and test the conjecture. Indeed the discovery of new protected operators was found by this method [33]. The information concerning operators in the OPE can be extracted from the four-point function by a conformal partial wave analysis (CPWA). This involves decomposing the correlator into contributions from different operators and was performed for the four-point correlator of energymomentum multiplets in $N=4 \mathrm{SYM}$ in $[39,43]$. A CPWA was performed for some higher charge half BPS operators in [41].

In [40] four-point functions of energy momentum multiplets and higher charge chiral primary operators were written down on analytic superspace. Higher charge four-point functions were also written down in [41] using a different method, and were compared with results from IIB supergravity on $A d S_{5} \times S^{5}$.

It is the purpose of this paper to apply analytic superspace techniques to look at the theory of six-dimensional $(2,0)$ tensor supermultiplets. In [12] this theory was considered in the harmonic superspace description, half BPS operators were identified as representations and correlation functions of half-BPS operators found. Using analytic superspace (closely related to harmonic superspace) we will extend these results and show how to find $n$-point correlation functions of any representations.

It is of particular interest to consider the four-point function of energy-momentum multiplets in the six-dimensional theory and to perform a CPW analysis on this. When considering the fourpoint functions in $N=4 \mathrm{SYM}$ it proved useful to expand in terms of Schur polynomials [40]. We will find that in the six-dimensional case the Schur polynomial is also useful for finding a simple expression for the correlator but one needs an expansion in a different basis in order to relate this to the CPW. As for the Schur polynomials the second basis has an interpretation in terms of supergroup representation theory. Furthermore, in the bosonic case this expansion reduces to the expansion in terms of Jack polynomials used in six-dimensional CFT in [44] and so we can view the supersymmetric expansion as the natural supersymmetric extension of these Jack polynomials.

The full superconformal partial wave expansion can be found by lifting from the bosonic case where the expansion is known [44]. Using this we are able to perform a CPWA on both the free theory and the large $\mathrm{N}$ theory which is dual to gauged supergravity via the AdS/CFT correspondence. In particular we find the first $1 / N^{3}$ corrections to the dimensions of all operators in the OPE of two energy-momentum multiplets. 
Finally we will consider operators in the theory which lie on the threshold of the a) unitary bounds according to the classification of unitary irreducible reps of [45] (see section B.3). In SYM operators lying on the $N=4$ superconformal series a) bound come in two types: those that develop anomalous dimensions and those that are protected. These protected operators were first noticed by analysing the four-point function of energy momentum tensors in the large $\mathrm{N}$ theory using AdS/CFT. This fact was later proved and generalised, firstly by analysing the four-point function of energy-momentum tensors [38], and then by analysing the Ward identities of three-point functions involving the operator in question and two half BPS operators [46]. The Ward identities give selection rules for the allowed dimensions of the operator in question implying that some of them must be protected.

In [26] another very simple proof was given which classifies an operator as protected or not based purely on whether it is short or not (respectively) in the classical interacting theory. In particular all operators which can be written in terms of half BPS operators and saturate the unitary a) bound is protected. Another recursive way to say this is that any operator which can be written in terms of protected operators and which saturates the unitary bounds is protected. Note that these operators need not lie in the OPE of two half BPS operators. It is however, presumably possible to prove their protectedness using the method of [46] by obtaining selection rules from three-point functions of more complicated operators than the half BPS ones. Indeed, as we shall see, in the six-dimensional case it turns out that one is forced to take this approach.

In the six-dimensional case there are two complications. Firstly the classical theory is not known and so the arguments of [26] can not be applied. Secondly the operators which lie on the threshold of the series a) bound do not lie in the OPE of two half BPS operators so the selection rule arguments of [46] can also not be applied straightforwardly [15]. We therefore consider selection rules obtained from analysing the three-point function of one half-BPS operator with one other protected (but not half BPS) operator and a third operator. We again find the existence of protected operators at the threshold of the unitary bounds.

The outline of the paper is as follows. In section 2 we introduce analytic superspace, consider the transformation of operators, the superconformal Ward identities and we show how to solve these Ward identities. In section 3 we examine the four-point function of energy momentum multiplets in detail outlining the main results from the rest of the paper. In section 4 we consider expanding an invariant four point function in Schur polynomials and Jack polynomials in a purely bosonic CFT in six dimensions in order to illustrate the techniques which we use in section 5 for the full superconformal case. In section 6 we perform a conformal partial wave analysis of the free four-point function and the AdS dual four-point function. In section 7 we consider how crossing symmetry acts on the four point function. In section 8 we rewrite the four-point function in a manner which allows more direct comparison with previous results and we discuss the relation with previous results. Finally in section 9 we discuss operators lying at the threshold of the unitary bound in the free theory. We find that some such operators are protected and remain short and we find representations with which others may combine to become long operators and hence develop anomalous dimensions. We leave to the appendix technical details concerning the construction of superspaces as supercosets of the superconformal group and how to find the transformation of operators. We also discuss the general construction of superconformal 
invariant $n$-point functions in the theory in the appendix.

Whilst this manuscript was in preparation the preprint [47] appeared which overlaps with the study of four-point functions performed here.

\section{Correlation functions}

The six-dimensional $(2,0)$ tensor supermultiplet consists of 5 scalar fields transforming under the fundamental representation of the internal group $S O(5) \sim U S p(4)$, two Majorana-Weyl spinors in the fundamental representation of $U s p(4)$, and a two form gauge field with a self-dual 3 -form field strength. In the free theory these components can be packaged together on analytic superspace into a single analytic superfield $W(X)[12]^{1}$. Analytic superspace has half the number of odd coordinates as ordinary $(2,0)$ Minkowski superspace, but also has an additional internal space $U(2) \backslash U S p(4)$. The local coordinates of analytic superspace combine into the $(4 \mid 2) \times(4 \mid 2)$ supermatrix

$$
X^{A B}=\left(\begin{array}{c|c}
x^{\alpha \beta} & \lambda^{\alpha b} \\
\hline-\left(\lambda^{T}\right)^{a \beta} & y^{a b}
\end{array}\right)
$$

where the $x$ s are the spacetime coordinates (in the spinor representation), the $y$ s are local coordinates on the internal space and the $\lambda \mathrm{s}$ are the odd coordinates. The indices $\alpha, \beta$ are 4-component spinor indices, $a, b$ are 2 component internal indices carrying the isotropy group $U(2)$ of the internal coset space. The matrix $X$ is generalised antisymmetric ${ }^{2}$ which means that $X^{A B}=-(-1)^{A B} X^{B A}$ and so

$$
x^{\alpha \beta}=-x^{\beta \alpha}=0 \quad y^{a b}=y^{b a} .
$$

Here $x^{\alpha \beta}=\left(\gamma_{a}\right)^{\alpha \beta} x^{a}$ is the spinor representation of the six-dimensional space-time coordinate $x^{a}$. Some properties of $\gamma$ matrices in six-dimensions are reviewed in appendix A.

Using supercoset techniques (see section B.2) it is straightforward to show that an infinitesimal superconformal transformation acts on these coordinates by

$$
\delta X=B+A X+X A^{s T}+X C X
$$

where $A, B, C$ are all $(4 \mid 2) \times(4 \mid 2)$ supermatrices, $B$ and $C$ are generalised anti-symmetric, and $A^{s T}$ denotes the supertranspose of the supermatrix $A$. The superfield $W$ transforms by

$$
\delta W=\mathcal{V} W+\Delta W
$$

where $\mathcal{V}$ is the vector field generating the transformation, $\delta X^{A B}=(\mathcal{V} X)^{A B}$ and

$$
\Delta:=\operatorname{str}(\mathrm{A}+\mathrm{XC}) .
$$

\footnotetext{
${ }^{1}$ Note here the close analogy with the case of four-dimensional $N=4$ SYM where the Yang-Mills multiplet can also be packaged into a single superfield on analytic superspace.

${ }^{2}$ We choose $\alpha$ to be an even index and $a$ to be an odd index so that for example generalised anti-symmetrisation of $A$ and $B$ corresponds to symmetrising $a$ and $b$ etc.
} 
More generally operators will have superindices carrying an irreducible representation of $\mathfrak{g l}(4 \mid 2)$, $\mathcal{R}$ (see section B.4) and they will transform as

$$
\delta \mathcal{O}_{\mathcal{R}}^{Q}=\mathcal{V} \mathcal{O}_{\mathcal{R}}^{Q}+\mathcal{R}(A(X)) \mathcal{O}_{\mathcal{R}}^{Q}+Q \Delta \mathcal{O}_{\mathcal{R}}^{Q}
$$

where $A(X)=A+X C$.

We can now write down and solve the Ward identities for correlators in the theory using similar techniques to those used for $N=4 \mathrm{SYM}$ in $[27,28]$. The Ward identities for a general correlation function

$$
<12 \ldots n>:=<\mathcal{O}_{\underline{A}_{1}}^{Q_{1}}\left(X_{1}\right) \ldots \mathcal{O}_{\underline{A}_{2}}^{Q_{n}}\left(X_{n}\right)>
$$

state that the correlator must be invariant under superconformal transformations. In other words

$$
\delta<12 \ldots n>=\sum_{i=1}^{n}\left(\mathcal{V}_{i}+\mathcal{R}_{i}\left(A_{i}\right)+Q_{i} \Delta_{i}\right)<12 \ldots n>=0
$$

where $A_{i}:=A\left(X_{i}\right) D_{i}:=D\left(X_{i}\right)$. These can be easily solved as follows.

For two-point functions we have the solution

$$
<\mathcal{T} \underline{A} \mathcal{O}_{\underline{A}}^{Q}(1) \mathcal{O}_{\underline{B}}^{Q}(2) \mathcal{T} \underline{B}>\propto\left(g_{12}\right)^{Q} \mathcal{T} \underline{A}\left(X_{12}^{-1}\right)_{\underline{A B}}^{n} \mathcal{T} \underline{B}
$$

where $\mathcal{O}_{\underline{A}}^{Q}$ is an operator in the representation $\mathcal{R}$ specified by a Young tableau with $n$ boxes which is carried by the multi-index $\underline{A}, \mathcal{T} \underline{A}$ is an arbitrary tensor also carrying the representation $\mathcal{R}$ and we define

$$
\left(X_{12}^{n}\right)_{\underline{A B}}:=\left(X_{12}\right)_{A_{1} B_{1}} \ldots\left(X_{12}\right)_{A_{n} B_{n}} .
$$

We have also introduced here the propogator $g_{12}$ which is the two point function of $W \mathrm{~s}$ in the free theory:

$$
<W(1) W(2)>\propto g_{12}=\operatorname{sdet}\left(X_{12}^{-1}\right)=\frac{\hat{y}_{12}^{2}}{x_{12}^{4}}
$$

where $X_{i j}:=X_{i}-X_{j}$ and where $\hat{y}=y+\lambda^{T} x^{-1} \lambda$.

The formula for the three-point function is as follows

$$
\begin{aligned}
\mathcal{T} \underline{A}_{1} \mathcal{T} \underline{A}_{2} \mathcal{T} \underline{A}_{3}<\mathcal{O}_{\underline{A}_{1}}^{Q_{1}} \mathcal{O}_{\underline{A}_{2}}^{Q_{2}} \mathcal{O}_{\underline{A}_{3}}^{Q_{3}}>\propto & \left(g_{12}\right)^{Q_{12}}\left(g_{23}\right)^{Q_{23}}\left(g_{31}\right)^{Q_{31}} \mathcal{T} \underline{A}_{1} \mathcal{T} \underline{A}_{2} \mathcal{T} \underline{A}_{3} \\
& \left(X_{12}^{-1}\right)_{\underline{A}_{2} \underline{B}_{2}}^{n_{2}}\left(X_{13}^{-1}\right)_{\underline{A}_{3} \underline{B}_{3}}^{n_{3}} \times t\left(X_{123}\right)_{\underline{A}_{1} \underline{B}_{3}}
\end{aligned}
$$

where $X_{123}=X_{12} X_{23}^{-1} X_{31}, Q_{i j}:=\frac{1}{2}\left(Q_{i}+Q_{j}-Q_{k}\right) ; k \neq i, j$ and where $t\left(X_{123}\right)$ is a monomial of $X_{123}$ and its inverse with the index structure as indicated. Note that in general $t$ is not unique and one must take a linear combination of terms (see section 9 for an example of this.)

Similar formulae can be found for the higher point functions and the general formula can be written schematically as ${ }^{3}$

$$
<12 . . n>=P \Pi_{j=2}^{n} \mathcal{R}_{j}\left(X_{1 j}^{-1}\right) \sum_{t} t_{\mathcal{R}_{1} \ldots \mathcal{R}_{1}^{\prime}}^{\mathcal{R}_{n} ; \mathcal{R}_{2}^{\prime} \ldots \mathcal{R}_{n}^{\prime}} F_{t}
$$

\footnotetext{
${ }^{3}$ see [28] for the analogous formula in $N=4 \mathrm{SYM}$
} 
Here the sum is over all possible tensors $t, P$ denotes an appropriate propogator factor, while $\mathcal{R}_{i}$ are the representations of the $\mathfrak{g l}(4 \mid 2)$ algebra under which the operators transform. Each $F_{t}$ is an arbitrary function of superconformal invariants which we discuss in appendix C. The correlator is further restricted by demanding analyticity in all internal variables. In the next section we discuss in detail the the four-point function of four energy-momentum multiplets.

\section{Four-point functions}

In [12] $W$ was used to generate a family of superfields in the free theory $A_{p}:=W^{p}$ which were shown to be superconformal. We wish to consider four-point functions of the supercurrent $T:=A_{2}=W^{2}$

$$
<T\left(X_{1}\right) T\left(X_{2}\right) T\left(X_{3}\right) T\left(X_{4}\right)>\text {. }
$$

Although we do not know the interacting theory we can still examine the Ward identities for correlation functions in the theory.

The superconformal Ward identities are

$$
\sum_{i=1}^{4}\left(\mathcal{V}_{i}+2 \Delta_{i}\right)<T T T T>=0
$$

The four-point function of four energy-momentum multiplets solving these Ward identities can be written on analytic superspace as

$$
<T T T T>=\left(g_{12} g_{34}\right)^{2} \times \mathcal{I}
$$

where $\mathcal{I}\left(X_{1}, X_{2}, X_{3}, X_{4}\right)$ is invariant under superconformal transformations. The invariant is further restricted by insisting that it is analytic in the internal coordinates. This is because $T$ is a polynomial in $y$ and hence analytic in $y$ so the right-hand side of (16) must be also.

We consider the problem of finding such a function $\mathcal{I}$. The analysis begins similarly to the four dimensional case which can be found in $[6,28]$. Translation invariance $\delta X_{i}=B$ requires the function to depend only on difference variables $X_{i j}$. Invariance under $C$ requires the function to depend only on the differences $X_{i j}^{-1}-X_{i k}^{-1}$ ie only on the variables $X_{i j k}:=X_{i j} X_{j k}^{-1} X_{k i}$ which transforms as

$$
\delta X_{i j k}=A_{i} X_{i j k}+X_{i j k} A_{i}^{s T}
$$

where $A_{i}:=A+X_{i} C$ (the variables $X_{i j k}$ are in fact the negative inverses of the differences $\left.X_{i j}^{-1}-X_{i k}^{-1}\right)$. For the four-point function there are only two independent variables $X_{i j k}$ which we choose to be $X_{213}$ and $X_{243}$. Now change variables so that our four-point function depends on the two variables $X_{213}$ and

$$
Z:=-X_{213} X_{243}^{-1}=X_{21} X_{13}^{-1} X_{34} X_{42}^{-1} .
$$

These variables transform as

$$
\begin{aligned}
\delta X_{213} & =A_{2} X_{213}+X_{213} A_{2}^{s T} \\
\delta Z & =A_{2} Z-Z A_{2} .
\end{aligned}
$$


Note that $Z$ transforms in the adjoint representation of $\mathfrak{g l}(4 \mid 2)$. We use this residual symmetry (19) to set the variable $X_{123}$ to the following form

$$
X_{213}=K:=\left(\begin{array}{cc|c}
0 & 1 & 0 \\
-1 & 0 & 0 \\
\hline 0 & 0 & 1
\end{array}\right)
$$

and then the remaining symmetry preserving $K$ is

$$
\mathfrak{o s p}(2 \mid 4)=\left\{A_{2}: A_{2} K+K A_{2}^{s T}=0\right\} .
$$

So we have reduced the problem of finding a four-point function invariant under the conformal group to one of finding a function of $Z$ invariant under the adjoint of $\mathfrak{o s p}(2 \mid 4)$. The finite version of this is invariance under the adjoint action of the group $O S p(2 \mid 4)$,

$$
Z \mapsto G^{-1} Z G
$$

where $G \in O S p(2 \mid 4)$.

There are now two bases which prove useful in expanding the invariant function $\mathcal{I}$. Firstly we use the basis provided by $G L(4 \mid 2)$ Schur polynomials. Schur polynomials (also known as characters) are simply (super)traces of representations $S_{\mathcal{R}}(Z)=\operatorname{str}(\mathcal{R}(\mathrm{Z})$ ) where $\mathcal{R}$ denotes a finite dimensional irreducible representation of $G L(4 \mid 2)$ (and hence a finite dimensional representation of $O S p(2 \mid 4))$. The allowed representations $\mathcal{R}$ for $\langle T T T T\rangle$ (that is representations which give a correlation function which is analytic in all the internal variables) can be described by a $G L(4 \mid 2)$ Young tableau with only two rows. We arrive at the formula

$$
<T T T T>=\left(g_{12} g_{34}\right)^{2} \sum_{p, \mathcal{R}} C_{p, \mathcal{R}}(\operatorname{sdet} Z)^{p} S_{\mathcal{R}}(Z) .
$$

Analyticity in the internal variables of the four-point function puts restrictions on the allowed representations $\mathcal{R}$ and the values of $p$. This follows the similar argument in the four dimensional case [40]. Indeed sdet $Z$ has poles in $y_{12}$ and this gives the restriction $p \leq 2$. The term $S_{\mathcal{R}}$ may contain poles in $y_{13}$ however and in order to remove these we require that $p \geq r$ where $r$ is the number of rows of the Young tableau of $\mathcal{R}$. So we find that for representations with two rows we must have $p=2$, for those with 1 row we can have $p=1,2$ and for the trivial representation we may have $p=0,1,2$.

Using this basis we can write the correlator in an explicit form as follows. We use the remaining $O S p(2 \mid 4)$ symmetry $G$ to transform $Z$ and bring it into the diagonal form

$$
Z=\operatorname{diag}\left(X_{1}, X_{2}, X_{1}, X_{2} \mid Y_{1}, Y_{2}\right)
$$

So we can write the invariant four-point function in terms of the eigenvalues $X_{1}, X_{2}, Y_{1}, Y_{2}$. Note that the the fact that the $X_{i}$ eigenvalues repeat simply comes from the fact that $Z$ wqas constructed using antisymmetric supermatrices. We will give explicit formulae for the Schur polynomials in terms of these variables below, but for now we simply state that they can be 
used to show that the entire correlator can be written in terms of a single function of two variables in the form

$$
<T T T T>=\frac{\left(g_{13} g_{24}\right)^{2}}{\left(X_{1}-X_{2}\right)^{4}}\left(\boldsymbol{\Delta}\left(\mathcal{S} F\left(X_{1}, X_{2}\right)\right)+\mathcal{S}_{1}^{2} F\left(X_{1}, X_{1}\right)+\mathcal{S}_{2}^{2} F\left(X_{2}, X_{2}\right)\right)
$$

where $\mathcal{S}:=\left(X_{1}-Y_{1}\right)\left(X_{1}-Y_{2}\right)\left(X_{2}-Y_{1}\right)\left(X_{2}-Y_{2}\right)$ and $\mathcal{S}_{i}=\left(X_{i}-Y_{1}\right)\left(X_{i}-Y_{2}\right)$ and $\boldsymbol{\Delta}$ is defined in (63).

There is another basis for the invariant function, however, which allows us to more easily relate the four-point function to the operators appearing in the OPE of two energy momentum multiplets and hence to perform a conformal partial wave analysis. This second basis is given by

$$
T_{\mathcal{R}}(Z):=\mathcal{Y}_{\mathcal{R}}\left(K^{n}\right)_{\underline{A}}\left(W^{n}\right)^{\underline{A}}
$$

where $\underline{A}$ is a multi index put into the representation $\mathcal{R}$ (which is given by a Young tableau with $2 n$ boxes) using a suitably normalised Young operator $\mathcal{Y}_{\mathcal{R}}$. The Young operator is defined similarly to the standard purely bosonic case the only difference being that (anti-)symmetrisation is generalised. The normalisation will be defined implicitly later when we give explicit formulae for $T_{\mathcal{R}}$ (for example in (44)). Here $K$ is defined in (21) and $W$ is defined by $Z=-K W$. In the purely bosonic case (the supersymmetric case can be straightforwardly reduced the bosonic case simply by taking all superindices to be usual six-dimensional Weyl spinor indices, and hence ignoring the internal indices) the basis elements $T_{\mathcal{R}}(Z)$ reduce to the Jack polynomials (up to a factor) which were used in six dimensional conformal field theory in [44]. The allowed representations $\mathcal{R}$ are specified by Young tableaux with four rows with the first two rows of equal length and the second two rows of equal length. For this expansion one obtains a similar formula to $(24)$

$$
<T T T T>=\left(g_{12} g_{34}\right)^{2} \sum_{p, \mathcal{R}} C_{p, \mathcal{R}}(\operatorname{sdet} Z)^{p} T_{\mathcal{R}}(Z) .
$$

with $p \leq 2$ and $p \geq r / 2$ where $r$ is the number of rows of the Young tableau of $\mathcal{R}$. So we find that for representations with four rows we must have $p=2$, for those with 2 rows we can have $p=1,2$ and for the trivial representation we may have $p=0,1,2$.

This latter formula facilitates a conformal partial wave analysis of the four-point function. The OPE for two energy-momentum tensors $T$ is given by

$$
T(1) T(2)=\sum_{\mathcal{R}_{M N}} \frac{A_{T T \mathcal{O}}}{(-8)^{N} C_{\mathcal{O O}}}\left(g_{12}\right)^{2-\frac{q}{2}}\left(X_{12}^{M+N}\right) \underline{A} \mathcal{O}_{\underline{A}}^{q}(2)+\ldots
$$

The dots denote contributions of descendants of the primary fields $\mathcal{O}_{\underline{A}}^{q}$, $\underline{A}$ is a multi-index containing $2 M+2 N$ indices and $\left(X_{12}^{2 M+2 N}\right) \underline{A}:=X_{12}^{A_{1} A_{2}} \ldots X_{12}^{A_{2 N+2 M-1} A_{2 N+2 M}}$. The operator $\mathcal{O}_{\underline{A}}^{q}$ carries the tensor representation $\mathcal{R}$ by having $2 N+2 M$ indices symmetrised according to the Young tableau (with $2 N+2 M$ boxes, $M$ boxes in each of the first two rows and $N$ in each of the third and fourth rows see (43)) corresponding to $\mathcal{R}$. $C_{\mathcal{O O}}$ is the coefficient for the two point function of two operators $\mathcal{O}_{\underline{A}}^{q}$ defined in (32), $A_{T T \mathcal{O}}$ is the coefficient of the three-point function defined in (31) whilst the numerical factor is present in order to reconcile the above definition of the OPE with the ordinary one in the bosonic case see section 4.1. Each primary field $\mathcal{O}_{\underline{A}}^{q}$ in this expansion carries charge $q=d / 2-j / 2$ where $d$ is the dilation weight and $j$ is the spin quantum number of the superconformal representation under which the operator transforms. As seen in the previous section, in general an operator on analytic superspace will also be a 
tensor (or quasi-tensor) field (indicated by the multi-index $\underline{A}$ ) carrying $2 n$ superindices and will transform under finite-dimensional irreducible representations of the $G L(4 \mid 2)$ group which act on the superindices $A, B, \ldots$ All of the indices must be covariant (subscript) in order to be unitary and the allowed representations must be those available in the decomposition of $\left(X_{12}^{n}\right)^{A}$ into irreducible representations. Since the building blocks, $X_{12}^{A B}$, for the Young tableaux are antisymmetric (corresponding to a Young tableau with 1 column and 2 rows) this means the allowed Young tableaux must have their first two rows of equal length, and their second two rows of equal length (analysis of the dependence on the internal coordinates shows that the Young tableaux can have no more than $q$ rows.)

An important formula is that contribution of an operator $\mathcal{O}_{\mathcal{R}}^{q}$ to the four-point function has the form

$$
<T T T T>\sim \frac{\left(A_{T T \mathcal{O}}\right)^{2}}{C_{\mathcal{O O}}}\left(g_{12} g_{34}\right)^{2}(\operatorname{sdet} Z)^{q / 2} \sum_{\mathcal{R}^{\prime}} C_{\mathcal{R}^{\prime}} T_{\mathcal{R}^{\prime}}(Z)
$$

where we sum over all representations $\mathcal{R}^{\prime}$ which have a Young tableau with a valid form that contains the Young tableau of $\mathcal{R}$ (ie the Young tableau of $\mathcal{R}^{\prime}$ can be obtained by adding boxes to that of $\mathcal{R}$.) Here $A_{T T \mathcal{O}}$ is the coefficient of the 3 -point function $\langle T T \mathcal{O}\rangle$

$$
<T T \mathcal{O}^{q} \cdot \mathcal{T}>=(-3)^{N} A_{T T \mathcal{O}} g_{12}^{2-q / 2} g_{13}^{q / 2} g_{23}^{q / 2}\left(X_{312}^{-1}\right)_{\underline{A}} \mathcal{T} \underline{A}
$$

where $X_{312}=X_{31} X_{12}^{-1} X_{23}, C_{\mathcal{O O}}$ is the coefficient of the two point function of two operators $\mathcal{O}_{M N}^{q}$

$$
<\mathcal{O}_{\underline{A}}^{Q}(1) \mathcal{O}_{\underline{B}}^{Q}(2) \mathcal{T} \underline{B}>=(4 !)^{N} C_{\mathcal{O O}}\left(g_{12}\right)^{Q}\left(X_{12}^{-n}\right)_{\underline{A B}} \mathcal{T} \underline{B}
$$

and $\mathcal{T} \underline{B}$ is an arbitrary tensor carrying the same representation $\mathcal{R}_{M N}$ carried by the operator $\mathcal{O}_{\underline{A}}^{q}$. Indeed the first term in the expansion (30) can easily be verified. One performs an OPE (29) on the four-point function at points $X_{3}, X_{4}$ and keeps only leading order terms in $X_{34}$ and only contributions from the operator $\mathcal{O}_{\mathcal{R}}^{q}$ to obtain

$$
\begin{aligned}
<T T T T> & \sim \frac{A_{T T \mathcal{O}}}{C_{\mathcal{O O}}}\left(g_{34}\right)^{2-q / 2} \mathcal{Y}_{\mathcal{R}}\left(X_{34}^{M+N}\right)^{\underline{A}}<T T \mathcal{O}_{\underline{A}}^{q}>+\ldots \\
& \propto \frac{\left(A_{T T \mathcal{O}}\right)^{2}}{C_{\mathcal{O O}}}\left(g_{12} g_{34}\right)^{2}(\operatorname{sdet} Z)^{q / 2} \mathcal{Y}_{\mathcal{R}}\left(X_{34}^{M+N}\right)^{\underline{A}}\left(X_{312}^{-1}\right)_{\underline{A}}+\ldots \\
& \propto \frac{\left(A_{T T \mathcal{O}}\right)^{2}}{C_{\mathcal{O O}}}\left(g_{12} g_{34}\right)^{2}(\operatorname{sdet} Z)^{q / 2} T_{\mathcal{R}}(Z)+\ldots
\end{aligned}
$$

where in the second line we have used (31) and in the third line we have used the definition (27) and the fact that $T_{\mathcal{R}}\left(X_{321}^{-1} X_{34}\right)=\mathcal{T}_{\mathcal{R}}(Z)+\ldots$ where the dots represent terms of higher order in $X_{34}$.

We have thus obtained the first term in the expansion of (28) and hence motivated the appearance of the object $T_{\mathcal{R}}$ in relation to a conformal partial wave expansion. In fact as we shall see the numerical coefficients $C_{\mathcal{R}}$ in (28) can be found using the results of [44].

Furthermore, by relating the Schur polynomials to $T_{\mathcal{R}}$ and using the relation to the OPE (30) one finds that the single two-variable function $F\left(X_{1}, X_{2}\right)$ splits as follows:

$$
F\left(X_{1}, X_{2}\right)=\lambda G\left(X_{1}, X_{2}\right)+\frac{f\left(X_{1}\right)-f\left(X_{2}\right)}{\lambda}+\frac{g\left(X_{1}\right)-g\left(X_{2}\right)}{X_{1} X_{2} \lambda}+\frac{A}{X_{1}^{2} X_{2}^{2}}
$$

where $\lambda:=X_{1}-X_{2}$. Here $A$ is the contribution of the identity operator in the OPE, $g(X)$ gives the contributions of operators with $q=2$ (all are short), $f(X)$ has contributions from short 
operators with $q=4$ only and $G\left(X_{1}, X_{2}\right)$ has contributions from all operators with $q=4$ (short and long) $)^{4}$.

In summary, the four-point function of four energy-momentum multiplets can be written on analytic superspace in the simple closed form of (26) in terms of a single function of two variables. The contributions of different types of operators in the OPE of two T's can be isolated and using the results of [44] a complete CPWA can be performed. Indeed the conformal partial wave analysis is performed explicitly in section 6.1 for the free theory and in section 6.2 for the AdS dual theory.

\section{Purely bosonic case}

We will mainly be interested in the six-dimensional $(2,0)$ supersymmetric theory, but the formalism can be applied straightforwardly to the case of $(n, 0)$ supersymmetry for any $n$ including the purely bosonic case $n=0$. Therefore we firstly use this simpler case to illustrate the techniques. In the bosonic case the superindex $A$ becomes a $6 \mathrm{~d}$ spinor index $\alpha=(1,2,3,4)$, and the supercoordinates $X^{A B}$ of (1) become the usual six-dimensional coordinates $x^{\alpha \beta}$ in the spinor representation corresponding to the upper left block of $X$ in (1). The conformal group acts as in (6) if we regard all supermatrices to be $4 \times 4$ matrices corresponding to the upper left blocks of their supermatrix counterparts. A scalar field of dimension 1 transforms like $W$ in (4) and we consider the four-point function of fields with dimension 2 which we denote $T$ by analogy with the supersymmetric case. The four-point function reads $\langle T T T T\rangle=\left(g_{12} g_{34}\right)^{2} \times \mathcal{I}$ with $\mathcal{I}$ invariant under conformal transformations. We then consider the problem of finding $\mathcal{I}$ and following the arguments of equations (16-28) we arrive at a function of the matrix $Z=\operatorname{diag}\left(X_{1}, X_{2}, X_{1}, X_{2}\right)$ and two alternative expansions, the $T$ expansion or the $S$ Schur polynomial expansion which we now proceed to find explicit expressions for.

We wish to define the basis $T_{\mathcal{R}}(Z)$ in the bosonic case $\left(Z=\operatorname{diag}\left(\mathrm{X}_{1}, \mathrm{X}_{2}, \mathrm{X}_{1}, \mathrm{X}_{2}\right)\right.$ in this case. $)$ We firstly consider the simplest representation which is the antisymmetric representation $\mathcal{R}=$. The basis element corresponding to this representation is simply

$$
T_{\mathcal{R}}(Z)=C_{\alpha \beta} W^{\alpha \beta}=\operatorname{tr}(Z)=2 X_{1}+2 X_{2} .
$$

One can also find the basis elements corresponding to Young tableaux with two rows of equal length $m$

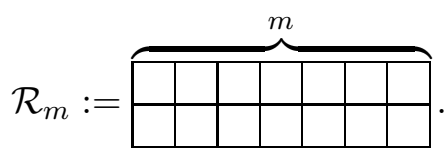

This is given by

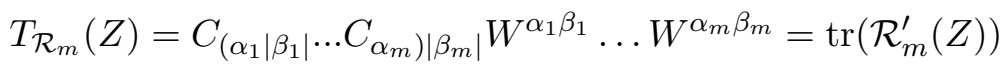

where we symmetrise over the $\alpha_{i}$ indices but not the $\beta_{i}$ indices. Here $\mathcal{R}_{m}^{\prime}$ is the completely symmetric representation with $m$ boxes and $\operatorname{tr}\left(\mathcal{R}^{\prime}(Z)\right)$ is simply the ordinary $G L(4)$ Schur

\footnotetext{
${ }^{4}$ We define a long operator to be one which can be given as a superfield on Minkowski superspace which has a full expansion in odd-coordinates with independent coefficients. A short operator is an operator which is not long.
} 
polynomial of $Z$. An explicit formula for these in terms of the eigenvalues $X_{1}, X_{2}$ can be found and is given by the formula

$$
t_{m}(Z):=\left(X_{1}-X_{2}\right)^{3} T_{\mathcal{R}_{m}}(Z)=(m+1)\left(X_{1}^{m+3}-X_{2}^{m+3}\right)-(m+3) X_{1} X_{2}\left(X_{1}^{m+1}-X_{2}^{m+1}\right)
$$

Although these are a priori only defined for $m \geq 0$ we will allow $m$ to take any integer value. We may then note the following important special cases

$$
t_{0}(Z)=1 \quad t_{-1}(Z)=t_{-2}(Z)=t_{-3}(Z)=0
$$

and also the relation

$$
t_{-m}(Z)=-\left(X_{1} X_{2}\right)^{2-m} t_{m-4}
$$

An explicit formula for the most general representation (occurring in the OPE of two scalars) is given by

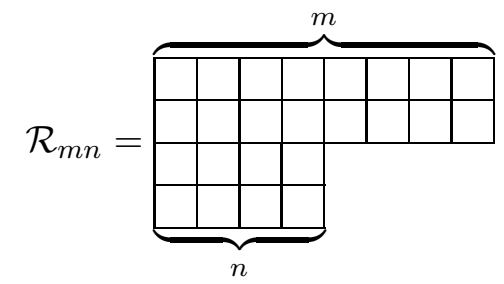

$$
\begin{aligned}
& \Downarrow \\
& t_{m n}(Z):=T_{\mathcal{R}_{m n}}\left(X_{1}-X_{2}\right)^{3}=\left(X_{1} X_{2}\right)^{n} t_{m-n}(Z) \\
& =(m-n+1) X_{1}^{m+3} X_{2}^{n}-(m-n+3) X_{1}^{m+2} X_{2}^{n+1}-\left(X_{1} \leftrightarrow X_{2}\right)
\end{aligned}
$$

Notice that the $T_{\mathcal{R}_{m}}$ are precisely (up to an overall factor) the Jack polynomials used in [44] in this context.

An invariant four-point function can be expanded in the basis $t_{m n}$. The relation (42) implies that

$$
t_{m-2 n}=-t_{n-2 m}
$$

and so although a priori $t_{m-2} n$ is only valid for $m-2 \geq n \geq 0$ (for the Young tableau (43)) to have the correct shape) we will extend this to any values in the range $m, n \geq 0$ by noting this symmetry (and also the fact that $t_{n-1, n}=t_{n-2, n}=t_{n-3, n}=0$.)

\subsection{Partial wave expansion}

In [44] the exact expression for the contribution of an operator in the OPE of two scalars to the four-point function of four such scalars in a purely bosonic six-dimensional conformal field theory was found as an expansion in Jack polynomials. This expression is known as the conformal partial wave and we briefly review this here for later use in the supersymmetric case. The formulae for the OPE and three-point functions given in $(29,31)$ can be applied straightforwardly to the purely bosonic case by simply letting all superindices become spinor indices and the supermatrix $X$ become the space-time variable $x$ in spinor notation. The energymomentum tensor $T$ becomes a scalar field $\phi(x)$ with dilation weight 2 and an operator $\mathcal{O}_{M, N}^{q}$ 
becomes isomorphic to an operator $\mathcal{O}_{M+q, N+q}$. We denote a general operator in the bosonic theory with dilation weight $M+N$ and spin $M-N$ by $\mathcal{O}_{M N}$. From now on in this section we set $q=0$ without loss of generality in the bosonic case. The isomorphism is given explicitly as

$$
\left(\mathcal{O}_{M N}^{0}\right)_{\underline{\alpha}}=\epsilon_{\alpha_{1} \alpha_{2} \alpha_{3} \alpha_{4}} \ldots \epsilon_{\alpha_{4 N-3} \ldots \alpha_{4 N}}\left(\mathcal{O}_{M N}\right)_{\alpha_{4 N+1} \ldots \alpha_{2 M+2 N}} .
$$

Here $\mathcal{O}_{M N}^{0}$ carries the tensor representation indicated by the Young tableau (43) and is therefore completely antisymmetric on the $N$ columns of the Young tableau which are therefore proportional to $\epsilon$ tensors as indicated. The operator $\mathcal{O}_{M N}$ has $2(M-N)$ spinor indices and is simply the spinor representation of a field with $M-N$ symmetric traceless space-time indices. The OPE (29) then reads

$$
\begin{aligned}
\phi\left(x_{1}\right) \phi\left(x_{2}\right) & \sim(-8)^{-N} A_{\phi \phi \mathcal{O}} C_{\mathcal{O O}}^{-1}\left(x_{12}^{2}\right)^{-4}\left(x_{12}^{M+N}\right)^{\underline{\alpha}}\left(\mathcal{O}_{M N}^{0}\right)_{\underline{\alpha}}(2)+\ldots \\
& =A_{\phi \phi \mathcal{O}} C_{\mathcal{O O}}^{-1}\left(x_{12}^{2}\right)^{N-4}\left(x_{12}^{M-N}\right)^{\underline{\alpha}}\left(\mathcal{O}_{M N}\right)_{\underline{\alpha}}(2)+\ldots
\end{aligned}
$$

This explains the presence of the factor $(-8)^{N}$ in (29) which is cancelled in (48) by the factor coming from the $N$ applications of (150).

If we similarly take the expression for the three point function (31) and define $\mathfrak{T}^{\underline{\alpha}}$, isomorphic to $\mathcal{T} \underline{\alpha}$, by

$$
\mathcal{T}^{\underline{\alpha}}=\epsilon^{\alpha_{1} \alpha_{2} \alpha_{3} \alpha_{4}} \ldots \epsilon^{\alpha_{4 N-3} \ldots \alpha_{4 N}} \mathcal{T}^{\alpha_{4 N+1} \ldots \alpha_{2 N+2 M}}
$$

then $\mathcal{O}_{M N}^{0} \cdot \mathcal{T}=(4 !)^{N} \mathcal{O}_{M N} \cdot \mathcal{T}$ and

$$
\begin{aligned}
\left\langle\phi \phi \mathcal{O}_{M N} \cdot \mathcal{T}\right\rangle & =(-8)^{-N} A_{\phi \phi \mathcal{O}}\left(x_{12}^{2}\right)^{-4}\left(x_{312}^{-1}\right)_{\underline{\alpha}}^{M+N} \mathcal{T}^{\underline{\alpha}} \\
& =A_{\phi \phi \mathcal{O}}\left(x_{12}^{2}\right)^{N-4}\left(x_{23}^{2}\right)^{-N}\left(x_{13}^{2}\right)^{-N}\left(x_{312}^{-1}\right)_{\underline{\alpha}}^{M-N} \mathcal{T}^{\underline{\alpha}} .
\end{aligned}
$$

Again we see that the numerical factor defined in (31) cancels.

We now consider the conformal partial wave expansion of the four-point function $<\phi \phi \phi \phi>$. Firstly consider this in the limit $x_{3} \rightarrow x_{4}$. To leading order in $x_{34}$ the contribution of the operator $\mathcal{O}_{M N}$ to this four-point function can be found by performing the OPE on $\phi(3) \phi(4)$ using (48) and then using (51). We obtain that the conformal partial wave expansion for the operator $\mathcal{O}_{M N}$ has the form

$$
\begin{aligned}
& \frac{A_{\phi \phi \mathcal{O}}}{C_{\mathcal{O O}}}<\phi \phi\left(\mathcal{O}_{M N}\right)_{\underline{\alpha}}>\left(x_{34}^{M-N}\right)^{\underline{\alpha}}\left(x_{34}^{2}\right)^{N-4}+\ldots \\
= & \frac{\left(A_{\phi \phi \mathcal{O}}\right)^{2}}{C_{\mathcal{O O}}} \frac{1}{\left(x_{12}^{2} x_{34}^{2}\right)^{4}}\left(z^{2}\right)^{N}\left(x_{312}^{-1}\right)_{\alpha_{1} \beta_{1}} \ldots\left(x_{312}^{-1}\right)_{\alpha_{M-N} \beta_{M-N}} x_{34}^{\left(\underline{\alpha}_{1}\left|\underline{\beta}_{1}\right|\right.} x_{34}^{\underline{\alpha}_{2}\left|\underline{\beta}_{2}\right|} \ldots x_{34}^{\left.\underline{\alpha}_{M-N}\right) \underline{\beta}_{M-N}}+\ldots \\
= & \frac{\left(A_{\phi \phi \mathcal{O}}\right)^{2}}{C_{\mathcal{O O}}} \frac{1}{\left(x_{12}^{2} x_{34}^{2}\right)^{4}} T_{\mathcal{R}_{M N}}(z)+\ldots
\end{aligned}
$$

In the second line we have to symmetrise the indices on the $x_{34} \mathrm{~s}$ as indicated and if we compare this with (39) we see the appearance of the Jack polynomial $T_{\mathcal{R}_{M-N}}$ which combines with the $\left(z^{2}\right)^{N}$ to give $T_{\mathcal{R}_{M N}}$ (see (44).) In all four of the equations above the dots indicate contributions from higher orders in $x_{34}$.

The full conformal partial wave (including all orders in $x_{34}$ ) corresponding to the operator $\mathcal{O}_{M N}$ was found in [44] and is given by

$$
<\phi(1) \phi(2) \phi(3) \phi(4)>\sim \frac{\left(A_{\phi \phi \mathcal{O}}\right)^{2}}{C_{\mathcal{O O}}} \frac{1}{\left(x_{12}^{2}\right)^{4}\left(x_{34}^{2}\right)^{4}} F_{M N}
$$


where $c$ is a constant and $F_{M N}$ is given by

$$
\begin{aligned}
F_{M N} & =\sum_{m, n \geq 0} c_{M, N}(m, n) T_{\mathcal{R}_{M+m, N+n}} \\
c_{M, N+2}(m, n) & =\frac{M-N-1}{\mu-1}\left(1-\frac{2 n}{(M-N-1)(\mu+1)}-\frac{2 m n}{(M-N-1)(\mu+1)(M+N)}\right) \frac{(M)_{m}^{2}}{(m) !(2 M)_{m}} \frac{(N)_{n}^{2}}{n !(2 N)_{n}}
\end{aligned}
$$

where $\mu:=M+m-N-n$. Notice that here we normalise $F_{M N}$ so that $c_{M, N}(0,0)=1$ and hence $F_{M N}=T_{\mathcal{R}_{M N}}+\ldots$ to be consistent with (54).

With this information, if we know the four-point function we can work out the coefficient $A_{\phi \phi \mathcal{O}}^{2} / C_{\mathcal{O O}}$ for all operators in the OPE. Furthermore, from one loop four-point functions one can find the anomalous dimensions of operators (see section 6.2).

\subsection{Schur polynomials}

As well as expanding the invariant four-point function in the Jack polynomials $T$ it is useful in the supersymmetric case to also consider an expansion in terms of Schur polynomials of $Z$ which we denote $S_{\mathcal{R}}(Z):=\operatorname{tr}(\mathcal{R}(Z)$ ). This latter expansion allows one to write the four point function in terms of a single two-variable function in the supersymmetric case.

In the bosonic case we know that (39)

$$
T_{\mathcal{R}_{m}}=S_{\mathcal{R}_{m}^{\prime}}
$$

So we already know the Schur polynomials for single row Young tableaux.

To find the Schur polynomials of more complicated Young tableaux we use the property that multiplication of Schur polynomials corresponds to the tensoring of the corresponding representations. In other words $s_{\mathcal{R}} s_{\mathcal{S}}=\sum_{\mathcal{T}} d_{\mathcal{R} \mathcal{S}} s_{\mathcal{T}}$ where $d_{\mathcal{R} \mathcal{S} \mathcal{T}}$ are the numbers in the decomposition of the tensor product of $\mathcal{S}$ and $\mathcal{R}$ into irreducibles: $\mathcal{R} \otimes \mathcal{S}=\sum_{\mathcal{T}} d_{\mathcal{R S} \mathcal{T}} \mathcal{T}$.

Using the well-known rules for multiplying Young tableaux one can show that $\mathcal{R}_{m n}^{\prime}=\mathcal{R}_{m}^{\prime} \mathcal{R}_{n}^{\prime}-$ $\mathcal{R}_{m+1}^{\prime} \mathcal{R}_{n-1}^{\prime}$ where
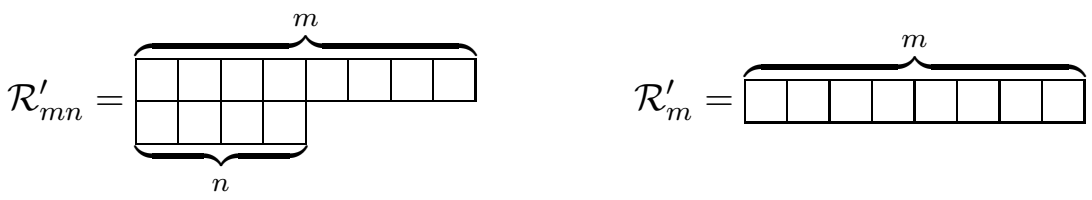

and it follows that

$$
S_{\mathcal{R}_{m n}^{\prime}}=S_{\mathcal{R}_{m}} S_{\mathcal{R}_{n}}-S_{\mathcal{R}_{m+1}} S_{\mathcal{R}_{n-1}}
$$

Explicitly, using $(44,57)$ one finds that

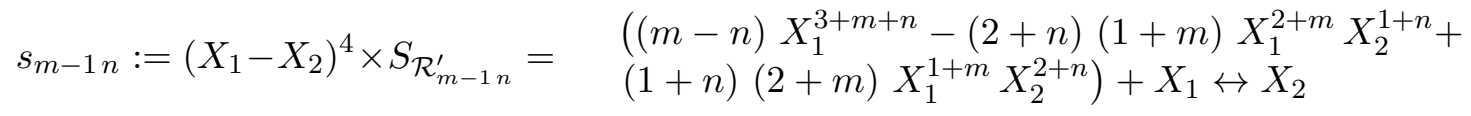

which satisfies:

$$
s_{m-1 n}=-s_{n-1 m} .
$$


So although $s_{m-1 n}$ is a priori only defined for $m-1 \geq n \geq 0$, one can extend it to the range $m, n \geq 0$ using the above equation and the fact that $s_{m m+1}=0$.

Equation (60) can be rewritten:

$$
\begin{aligned}
s_{m-1 n} & =\boldsymbol{\Delta}\left(\frac{X_{1}^{m+2} X_{2}^{n+2}-X_{1}^{n+2} X_{2}^{m+2}}{X_{1}-X_{2}}\right)+(m-n)\left(X_{1}^{3+m+n}+X_{2}^{3+m+n}\right) \\
\boldsymbol{\Delta} & :=-\left(\partial_{1}-\partial_{2}+\lambda \partial_{1} \partial_{2}\right) \lambda
\end{aligned}
$$

where $\lambda=X_{1}-X_{2}$.

One can expand a four-point function in the ' $\mathrm{s}$ ' basis as well as the 't' basis and this can be expressed in terms of an antisymmetric function of two variables $F$ as

$$
\begin{aligned}
\sum_{m, n \geq 0} d_{m n} s_{m-1 n}\left(X_{1}, X_{2}\right) & =\Delta F\left(X_{1}, X_{2}\right)+F\left(X_{1}, X_{1}\right)+F\left(X_{2}, X_{2}\right) \\
F\left(X_{1}, X_{2}\right) & :=\frac{1}{\lambda} \sum_{m, n \geq 0} d_{m n}\left(X_{1}^{m+2} X_{2}^{n+2}-X_{1}^{n+2} X_{2}^{m+2}\right)
\end{aligned}
$$

a formula which can be readily generalised to the supersymmetric case.

The ' $\mathrm{s}$ ' basis is related to the ' $\mathrm{t}$ ' basis by

$$
\lambda t_{m-2 n}=\frac{(m-n+1) s_{m-2 n}-(m-n-1) s_{m-1} n-1}{(m+1)(n+1)} .
$$

This formula generalises directly to the supersymmetric case where it is more useful than in the present context.

Before considering the supersymmetric case we would like to know to what extent the function $F\left(X_{1}, X_{2}\right)$ uniquely defines the four-point function. One can show that the general solution of $\boldsymbol{\Delta} F\left(X_{1}, X_{2}\right)+F\left(X_{1}, X_{1}\right)+F\left(X_{2}, X_{2}\right)=0, F\left(X_{1}, X_{2}\right)=F\left(X_{2}, X_{1}\right)$ is given by

$$
F\left(X_{1}, X_{2}\right)=\frac{f\left(X_{1}\right)-f\left(X_{2}\right)+X_{1} X_{2}\left(g\left(X_{1}\right)-g\left(X_{2}\right)\right)}{X_{1}-X_{2}}
$$

with $f, g$ arbitrary functions.

\section{$5 \quad$ The supersymmetric case}

\subsection{Operators in the OPE of two energy momentum multiplets}

Before we consider the four-point function of four energy momentum multiplets $T$ in the $(2,0)$ supersymmetric case, we first classify the operators which will appear in the OPE of two Ts and hence in the CPW expansion of the four-point function.

The starting point for the classification of operators is the formula for the OPE given in equation (29) and the analysis sketched below that equation shows that operators in the OPE must 
carry representations of $G L(4 \mid 2)$ given by the Young tableaux:

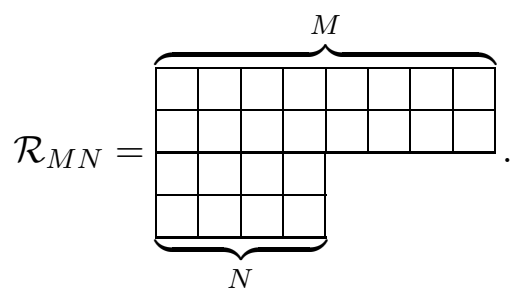

Notice that these Young tableaux are the same as those defining operators in the bosonic OPE (see (43)) although of course the interpretation is different: in the bosonic case one is considering $G L(4)$ representations whereas here we are considering $G L(4 \mid 2)$ representations. However this point turns out to be crucial when considering the CPW expansion since it enables us to read off the supersymmetric CPW from the bosonic one. In the supersymmetric case the operators also carry a charge $q=0,2$ or 4 and the Young tableau can have no more than $q$ rows.

The representations of $G L(4 \mid 2), \mathcal{R}_{M N}$ split into four classes: $N \geq 2$ are long representations (called typical in the mathematics literature) and lead to long supermultiplets ${ }^{5} ; N=1$ gives one class of short (or atypical) representations; $N=0, M \neq 0$ gives another class of short representations; finally $M=N=0$ is the trivial representation. We denote a general operator carrying charge $q$ and $G L(4 \mid 2)$ representation $\mathcal{R}_{M N}$ by $\mathcal{O}_{M N}^{q}$. We denote component fields which carry a representation of the internal group $U S p(4)$ as well as Lorentz spin $M-M$ and dilation weight $M+M$ by $\varphi_{M, N}^{\text {rep }}$ where rep is the dimension of the $U S p(4)$ representation. There are seven classes of operators, given in table 1 along with the lowest component of the multiplet and the component field obtained in taking the 'bosonic limit' which we do in section 5.3 in order to find the superconformal partial wave expansion.

\begin{tabular}{|lll|}
\hline Superfield & $\begin{array}{l}\text { Lowest } \\
\text { component }\end{array}$ & $\begin{array}{l}\text { 'Bosonic limit' } \\
\text { component }\end{array}$ \\
\hline $\mathcal{O}_{M, N \geq 2}^{4}$ & $\varphi_{M+2, N+2}^{\mathbf{1}}$ & $\varphi_{M+4, N+4}^{\mathbf{5 5}}$ \\
$\mathcal{O}_{M, 1}^{4}$ & $\varphi_{M+2,4}^{\mathbf{1 0}}$ & $\varphi_{M+4,5}^{\mathbf{5 5}}$ \\
$\mathcal{O}_{M, 0}^{4}$ & $\varphi_{M+2,4}^{\mathbf{1 4}}$ & $\varphi_{M+4,4}^{\mathbf{5 5}}$ \\
$\mathcal{O}_{0,0}^{4}$ & $\varphi_{4,4}^{\mathbf{5 5}}$ & $\varphi_{4,4}^{\mathbf{5 5}}$ \\
$\mathcal{O}_{M, 0}^{2}$ & $\varphi_{M, 2}^{\mathbf{1}}$ & $\varphi_{M+2,2}^{\mathbf{1 4}}$ \\
$\mathcal{O}_{0,0}^{2}$ & $\varphi_{2,2}^{\mathbf{1 4}}$ & $\varphi_{2,2}^{\mathbf{1 4}}$ \\
$\mathcal{O}_{0,0}^{0}$ & $\varphi_{0,0}^{\mathbf{1}}$ & $\varphi_{0,0}^{\mathbf{1}}$ \\
\hline
\end{tabular}

Table 1: Operators in the OPE of two Ts.

\subsection{The invariant four-point function in the supersymmetric case}

We now consider the four-point function of four energy-momentum multiplets in the $(2,0) \mathrm{su}-$ persymmetric theory. Remarkably much of the formalism from the bosonic case goes through fairly straightforwardly in the supersymmetric case also.

\footnotetext{
${ }^{5}$ In fact the long representations can have non-integer $n$ a fact which allows long operators to develop anomalous dimensions. For this one must use quasi-tensor representations [27]. This will not concern us in the present work.
} 
Firstly we wish to find formulae for the basis elements $T_{\mathcal{R}}$ with which we will expand the four-point function as in (28). The simplest representation is the (generalised) anti-symmetric representation $\mathcal{R}=$ G. The basis element corresponding to this representation is

$$
T_{\mathcal{R}}(Z)=K_{\underline{A B}} W \underline{A B}=\operatorname{tr}(Z)=2 X_{1}+2 X_{2}-Y_{1}-Y_{2} .
$$

As in the bosonic case, the basis elements $T_{\mathcal{R}}$ of Young tableaux with two rows of equal length $m$ coincide with the $G L(4 \mid 2)$ Schur polynomials of Z, $\operatorname{str}\left(\mathcal{R}_{\mathrm{m}}^{\prime}(\mathrm{Z})\right.$ ), of representations with a single row (see eq (39)). These can be found explicitly by decomposing the supergroup $G L(4 \mid 2)$ into its maximal bosonic subgroup $G L(4) \times G L(2)$.

The Schur polynomials respect this decomposition in the following sense. If the $G L(4 \mid 2)$ representation $\mathcal{R}$ decomposes as $\mathcal{R} \rightarrow \sum_{\mathcal{S}, \mathcal{T}} d_{\mathcal{R S} \mathcal{T}} \mathcal{S} \otimes \mathcal{T}$ under $G L(4 \mid 2) \supset G L(4) \times G L(2)$ (here $\mathcal{S}$ is a $G L(4)$ representation and $\mathcal{T}$ a $G L(2)$ representation and $\mathcal{S} \otimes \mathcal{T}$ is hence a representation of $G L(4) \times G L(2) \subset G L(4 \mid 2))$ then the Schur polynomials satisfy $s_{\mathcal{R}}(Z)=\sum_{\mathcal{S}, \mathcal{T}} d_{\mathcal{R S T}} s_{\mathcal{S}}(X) s_{\mathcal{T}}(Y)$ where

$$
Z=\left(\begin{array}{c|c}
X & 0 \\
\hline 0 & Y
\end{array}\right)
$$

Using this one can write the supersymmetric Schur polynomials in terms of the bosonic Schur polynomials. For example the Schur polynomials with only one row in their Young tableaux are given by:

$$
S_{m}(Z)=s_{m}-s_{m-1}\left(Y_{1}+Y_{2}\right)+s_{m-2} Y_{1} Y_{2} .
$$

This formula is a priori only valid for $m \geq 2$ since $s_{m}$ is only valid for $m \geq 0$. However from (41) we see that the formula also gives the correct answer for $S_{1}$ and $S_{0}$ without modification and also gives a vanishing $S_{-1}$

$$
S_{0}(Z)=1 \quad S_{-1}(Z)=0 .
$$

Note that these relations distinguish the six dimensional case from the case of four dimensional $N=4$ super Yang-Mills where Schur polynomials for short representations had to be treated separately [40]. This in turn leads to the requirement of extra functions of one variable in the four-dimensional case whereas one only needs a single two variable function in the sixdimensional case.

Similarly for Schur polynomials with two rows $S_{m n}:=\lambda^{4} S_{\mathcal{R}_{m n}^{\prime}}$ decomposes naturally into its component purely bosonic Schur polynomials:

$$
\begin{aligned}
S_{m-1 n}\left(X_{1}, X_{2}, Y_{1}, Y_{2}\right)= & s_{m-1 n}-\left(Y_{1}+Y_{2}\right)\left(s_{m-1 n-1}+s_{m-2 n}\right)+ \\
& Y_{1} Y_{2}\left(s_{m-1 n-2}+s_{m-2 n-1}+s_{m-3 n}\right)+\left(Y_{1}^{2}+Y_{1} Y_{2}+Y_{2}^{2}\right) s_{m-2 n-1}- \\
& Y_{1} Y_{2}\left(Y_{1}+Y_{2}\right)\left(s_{m-2 n-2}+s_{m-3 n-1}\right)+Y_{1}^{2} Y_{2}^{2} s_{m-3 n-2}
\end{aligned}
$$

and using (61) we find that

$$
S_{m-1 n}=-S_{n-1 m}
$$

and so we can extend the range of validity of $S_{m n}$ to $m \geq-1, n \geq 0$.

Equation (73) can be rewritten (using (62)) in the form

$$
\begin{aligned}
S_{m-1 n}=\Delta\left(\mathcal{S} \times\left(\frac{X_{1}^{m} X_{2}^{n}-X_{1}^{n} X_{2}^{m}}{X_{1}-X_{2}}\right)\right) \begin{array}{l}
+(m-n)\left(X_{1}-Y_{1}\right)^{2}\left(X_{1}-Y_{2}\right)^{2} X_{1}^{m+n-1} \\
+(m-n)\left(X_{2}-Y_{1}\right)^{2}\left(X_{2}-Y_{2}\right)^{2} X_{2}^{m+n-1}
\end{array}
\end{aligned}
$$




$$
\begin{aligned}
\mathcal{S}:= & X_{1}^{2} X_{2}^{2}-\left(Y_{1}+Y_{2}\right) X_{1} X_{2}\left(X_{1}+X_{2}\right)+Y_{1} Y_{2}\left(X_{1}^{2}+X_{1} X_{2}+X_{2}^{2}\right)+ \\
& Y_{1}^{2} Y_{2}^{2}-\left(Y_{1}+Y_{2}\right) Y_{1} Y_{2}\left(X_{1}+X_{2}\right)+X_{1} X_{2}\left(Y_{1}^{2}+Y_{1} Y_{2}+Y_{2}^{2}\right) . \\
= & \left(X_{1}-Y_{1}\right)\left(X_{1}-Y_{2}\right)\left(X_{2}-Y_{1}\right)\left(X_{2}-Y_{2}\right)
\end{aligned}
$$

It is also useful to note the two identities

$$
\begin{aligned}
S_{m,-1} & =\frac{Y_{1} Y_{2}}{\left(X_{1} X_{2}\right)^{2}} S_{m+10} \\
S_{-2,-2} & =-S_{-3,-1}=\frac{\left(Y_{1} Y_{2}\right)^{2}}{\left(X_{1} X_{2}\right)^{4}} .
\end{aligned}
$$

which are crucial for allowing us to treat the contributions of operators of dimension 2 and the identity operator in the same formula.

Therefore an invariant four-point function $\mathcal{I}$ expanded in the ' $\mathrm{S}$ ' basis can be written in terms of a single function of two variables $F\left(X_{1}, X_{2}\right)$ as

$$
\begin{aligned}
\lambda^{4} \mathcal{I}=\mathcal{F}[F]:=\sum_{m, n \geq 0} f_{m n} S_{m-1 n} & =\boldsymbol{\Delta}\left(\mathcal{S} F\left(X_{1}, X_{2}\right)\right)+\mathcal{S}_{1}^{2} F\left(X_{1}, X_{1}\right)+\mathcal{S}_{2}^{2} F\left(X_{2}, X_{2}\right)(80) \\
F\left(X_{1}, X_{2}\right) & =\sum_{m, n \geq 0} f_{m n} \frac{X_{1}^{m} X_{2}^{n}-X_{2}^{m} X_{1}^{n}}{X_{1}-X_{2}}
\end{aligned}
$$

where $\mathcal{S}_{i}=\left(X_{i}-Y_{1}\right)\left(X_{i}-Y_{2}\right)$. This is the complete result for the four-point function of four energy-momentum multiplets given in (26).

In order to separate out correctly the contributions from short and long operators to the fourpoint function, equation (30) tells us we need to use the $T$ basis rather than the $S$ basis. In order to find explicit expressions for the $T$ basis we note that it is related to the Schur polynomial $S$ basis by a similar formula to the bosonic case (see (66))

$$
\lambda T_{m-2 n}= \begin{cases}\frac{(m-n+1) S_{m-2 n}-(m-n-1) S_{m-1} n-1}{(m+1)(n+1)} & m, n \geq 1 \\ S_{m-20} & n=0\end{cases}
$$

In the supersymmetric case (unlike the bosonic case) $n=0$ has to be handled seperately since our expression for $S_{m n}$ (73) does not give $S_{m-1}=0$. From (74) we find that this expression satisfies the symmetry

$$
T_{m-2 n}=-T_{n-2 m}
$$

which can be used to extend the range of validity of (82a) from $m-2 \geq n \geq 1$ to the range $n \geq 1, m \geq 1$.

Equation (82) can be rewritten, using $(75,44)$ as

$$
\begin{aligned}
& \begin{array}{ll}
\lambda T_{m-2 n}=-\Delta\left(\frac{S}{\lambda X_{1} X_{2}} \frac{t_{m-2 n}}{(m+1)(n+1)}\right) & m, n \geq 1
\end{array} \\
& \begin{aligned}
\lambda T_{m-20}=\boldsymbol{\Delta}\left(S \frac{X_{1}^{m-1}-X_{2}^{m-1}}{\lambda}\right) & +(m-1)\left(X_{1}-Y_{1}\right)^{2}\left(X_{1}-Y_{2}\right)^{2} X_{1}^{m-2} \\
& +(m-1)\left(X_{2}-Y_{1}\right)^{2}\left(X_{2}-Y_{2}\right)^{2} X_{2}^{m-2}
\end{aligned} \\
& =-\boldsymbol{\Delta}\left(\frac{S}{\lambda X_{1} X_{2}} \frac{t_{m-2} 0}{(m+1)}\right)+\frac{m-1}{m+1} S_{m-1,-1}
\end{aligned}
$$

We also use the following which comes from $(75,78,79,82 \mathrm{~b})$

$$
\begin{aligned}
\lambda \frac{Y_{1} Y_{2}}{X_{1}^{2} X_{2}^{2}} T_{m-20}=S_{m-3,-1} & \\
=\Delta\left(\frac{S}{X_{1} X_{2}} \frac{X_{1}^{m-1}-X_{2}^{m-1}}{\lambda}\right) & +(m-1)\left(X_{1}-Y_{1}\right)^{2}\left(X_{1}-Y_{2}\right)^{2} X_{1}^{m-4} \\
& +(m-1)\left(X_{2}-Y_{1}\right)^{2}\left(X_{2}-Y_{2}\right)^{2} X_{2}^{m-4}
\end{aligned}
$$


The contributions of the last two terms of (75) vanish for $m, n \geq 1$.

The invariant function $\mathcal{I}$ needed in the four-point function expanded in the ' $\mathrm{T}$ ' basis consists of four terms all of which can be written in the form (80) in terms of a function of two variables in the following way.

$$
\lambda \sum_{m, n \geq 1} d_{m n} T_{m-2} n+\lambda \sum_{m \geq 0} d_{m} T_{m 0}+\lambda \frac{Y_{1} Y_{2}}{X_{1}^{2} X_{2}^{2}} \sum_{m \geq 0} c_{m} T_{m 0}+A \frac{Y_{1}^{2} Y_{2}^{2}}{X_{1}^{4} X_{2}^{4}}=\mathcal{F}\left[F_{1}+F_{2}+F_{3}+F_{4}\right]
$$

where

$$
\begin{aligned}
& F_{1}=\lambda G \\
& F_{2}=\left(f\left(X_{1}\right)-f\left(X_{2}\right) / \lambda\right. \\
& F_{3}=\left(g\left(X_{1}\right)-g\left(X_{2}\right) /\left(X_{1} X_{2} \lambda\right)\right. \\
& F_{4}=A /\left(X_{1}^{2} X_{2}^{2}\right)
\end{aligned}
$$

The split into $F_{1}, F_{2}, F_{3}, F_{4}$ is unique if we assume holomorphicity of all functions. In this way, one can isolate the contributions of short operators in the OPE.

An important point to note is that in the supersymmetric case, by writing the four-point function in the form $\mathcal{F}[F]$ as in (80), the function $F$ is uniquely defined. Indeed using (67) one can show that the solution of $\mathcal{F}[F]=0$ for all values of $Y_{1}, Y_{2}$ is $F=0$.

\subsection{Superconformal partial wave expansion}

We are now in a position to give a complete superconformal partial wave expansion for the four-point function of four energy-momentum multiplets. In order to do this we consider the four-point function $<T T T T>$ in the limit where $y_{12}, y_{34} \rightarrow 0$ and where all analytic superspace odd coordinates vanish. We call this the 'bosonic limit'. In this limit the remaining symmetry is the $d=6$ conformal subgroup of the full superconformal group. Furthermore, the variables $Y_{1}, Y_{2} \rightarrow 0$ and the supersymmetric polynomial $T_{m n} \rightarrow t_{m n}$ and since conformal symmetry is still present, the superconformal partial wave expansion of the superspace operator $\mathcal{O}$ reduces to the (non-supersymmetric) conformal partial wave expansion of a component field of $\mathcal{O}$. One finds that the superconformal partial wave corresponding to $\mathcal{O}_{\mathcal{R}_{M N}}^{4}$ becomes the conformal partial wave of $\varphi_{M+4, N+4}^{\mathbf{5 5}}$ (an operator in the $\mathbf{5 5}$ representation of $U S p(4)$, with Lorentz spin $j=M-N$ and dilation weight $D=M+N+8$ ) and the superconformal partial wave corresponding to $\mathcal{O}_{M, 0}^{2}$ becomes the conformal partial wave of the component $\varphi_{M+2,2}^{\mathbf{1 4}}$ (see table 1).

Explicitly then, the superconformal partial wave expansion of the operator $\mathcal{O}_{\mathcal{R}_{M N}}^{4}$

$$
<T T T T>\sim A_{M N}\left(g_{12} g_{34}\right)^{2}(\operatorname{sdet} Z)^{2} \sum_{m, n} C_{M N}^{4}(m, n) \frac{T_{M+m, N+n}}{\lambda^{3}}
$$

in this limit becomes

$$
\begin{aligned}
<T T T T> & \sim A_{M N}\left(y_{13}^{2}\right)^{4}\left(x_{12}^{2}\right)^{-4}\left(x_{34}^{2}\right)^{-4} \sum_{m, n} C_{M N}^{4}(m, n) \frac{t_{M+4+m, N+4+n}}{\lambda^{3}} \\
& \sim A_{M N}\left(y_{13}^{2}\right)^{4}\left(x_{12}^{2}\right)^{-4}\left(x_{34}^{2}\right)^{-4} F_{M+4, N+4}
\end{aligned}
$$


see $(55,56)$. This gives

$$
C_{M N}^{4}(m, n)=c_{M+4, N+4}(m, n)
$$

where $c_{M N}(m, n)$ are the coefficients defined in (56) for the bosonic conformal partial wave expansion. Similarly the superconformal partial wave expansion of the operator $\mathcal{O}_{\mathcal{R}_{M 0}}^{2}$

$$
<T T T T>\sim B_{M}\left(g_{12} g_{34}\right)^{2} \operatorname{sdet} Z \sum_{m, n} C_{M}^{2}(m, n) \frac{T_{M+m, n}}{\lambda^{3}}
$$

becomes

$$
\begin{aligned}
\left(y_{12}\right)^{-2}\left(y_{34}\right)^{-2}<T T T T> & \sim B_{M}\left(y_{13}\right)^{4}\left(x_{12}\right)^{-8}\left(x_{34}\right)^{-8} \sum_{m, n} C_{M}^{2}(m, n) \frac{t_{M+2+m, 2+n}}{\lambda^{3}} \\
& \sim B_{M}\left(y_{13}\right)^{8}\left(x_{12}\right)^{-8}\left(x_{34}\right)^{-8} F_{M+2,2}
\end{aligned}
$$

where $F_{M N}$ is given in (56) giving

$$
C_{M}^{2}(m, n)=c_{M+2,2}(m, n) .
$$

In this way we have found the complete superconformal partial wave expansion for $\langle T T T T\rangle$.

Note that the coefficients $A_{M N}$ and $B_{M}$ give the following combination of the three-point function coefficient and the two-point function coefficient as defined in $(31,32)$

$$
\frac{\left(A_{T T \mathcal{O}}\right)^{2}}{C_{\mathcal{O O}}}
$$

It is possible to write the conformal partial wave expansion in the form (80) in terms of the functions $G, f, g$ defined in (87). For example, by inserting (95) into (92) and comparing with (86) we find that the operator $\mathcal{O}_{M 0}^{2}$ contributes $G=f=0$ and

$$
\mathcal{O}_{M 0}^{2} \quad \rightarrow \quad g(X) \sim X^{M+1} F_{21}(M+2, M+1 ; 2 M+4 ; X):=g_{M}(X)
$$

where $F_{21}$ is a hypergeometric function.

\section{Superconformal partial wave analysis of the free and large $\mathrm{N}$ four point function.}

\subsection{The free four-point function.}

The free four-point function has the following form:

$$
\begin{aligned}
<T T T T>=g_{13}^{2} g_{24}^{2} & \left(A\left(1+\operatorname{sdet}(Z)^{-2}+\operatorname{sdet}(1-Z)^{-2}\right)\right. \\
& \left.+B\left(\operatorname{sdet}(Z)^{-1}+\operatorname{sdet}(1-Z)^{-1}+\operatorname{sdet}(Z)^{-1} \operatorname{sdet}(1-Z)^{-1}\right)\right) .
\end{aligned}
$$

One can expand the superdeterminants in terms of Schur polynomials and thus write this in the form (87):

$$
\begin{aligned}
<\mathrm{TTTT}> & =\lambda^{-4} g_{13}^{2} g_{24}^{2} \mathcal{F}[F] \\
F\left(X_{1}, X_{2}\right) & =A\left(1+\frac{1}{X_{1}^{2} X_{2}^{2}}+\frac{1}{\left(1-X_{1}\right)^{2}\left(1-X_{2}\right)^{2}}\right) \\
& +B\left(\frac{1}{X_{1} X_{2}}+\frac{1}{\left(1-X_{1}\right)\left(1-X_{2}\right)}+\frac{1}{X_{1} X_{2}\left(1-X_{1}\right)\left(1-X_{2}\right)}\right)
\end{aligned}
$$


Decomposing $F$ into $G, f, g, A$ according to (87), we obtain

$$
\begin{aligned}
G\left(X_{1}, X_{2}\right) & =-\frac{A\left(X_{1}-X_{2}\right)}{3\left(1-X_{1}\right)^{3}\left(1-X_{2}\right)^{3}} \\
f(X) & =A\left(X+\frac{1}{3(1-X)^{3}}\right)+B \frac{X}{1-X} \\
g(X) & =B\left(X+\frac{X}{1-X}\right) \\
A & =A
\end{aligned}
$$

We can now expand these functions in terms of the conformal partial waves calculated in the previous section. Using a computer one can check that

$$
g(X)=B(X+X /(1-X))=\sum_{j=0}^{\infty} B_{M} g_{M}(X)
$$

where

$$
B_{M}= \begin{cases}B \frac{(M+2) !(M) !}{(2 M+1) !} & M \text { even } \\ 0 & M \text { odd }\end{cases}
$$

and $g_{M}(X)$ are the conformal partial waves found in (97).

The operator $\mathcal{O}_{M 0}^{4}$ contributes $g=0$ and

$$
f(X) \sim \frac{\mathrm{d}^{2}}{\mathrm{dx}^{2}}\left(\frac{X^{M+3} F_{21}(M+1, M+4,2 M+8, X)}{(M+2)(M+3)}\right):=f_{M}(X) .
$$

In the free theory

$$
f(X)=A\left(X+\frac{1}{3(1-X)^{3}}-\frac{1}{3}\right)+B \frac{X}{1-X}=\sum_{j=0}^{\infty} A_{M 0} f_{M}(X)
$$

where

$$
A_{M 0}= \begin{cases}\frac{A(2+M)(3+M) !(6+M) !}{36(5+2 M) !}+\frac{B(2+M)^{2}(M) !(5+M) !}{(4+M)(5+2 M) !} & M \text { even } \\ 0 & M \text { odd }\end{cases}
$$

Finally one may sum up the contribution (given in (88)) of all operators $\mathcal{O}_{M N}^{4}$ to $G\left(X_{1}, X_{2}\right)$ (with the help of (87)) and compare with the free theory. One has to solve the linear equations

$$
\sum_{N=0}^{\infty} \sum_{M=N}^{\infty} \sum_{m=0}^{\infty} \sum_{n=0}^{\infty} c_{M+4, N+4}(m, n) A_{M N} \frac{t_{M+m, N+n}\left(X_{1}, X_{2}\right)}{X_{1} X_{2}(M+m+3)(N+n+1)}=\lambda^{2} G\left(X_{1}, X_{2}\right)
$$

where $A_{M 0}$ is given above. These equations can be solved order by order. Again using a computer we can show that the coefficients $A_{M N}$ are consistent with the formula

$$
A_{M N}= \begin{cases}\frac{(M+3) !(M+4) !}{(2 M+5) !} \frac{(N+1) !(N+2) !}{(2 N+1) !}(M-N+2)(M+N+5) \times \\ \left(\frac{A}{72}(M+N+6)+(-1)^{N} \frac{B}{2} \frac{1}{(M-N+1)(M-N+3)(M+N+4)}\right) & M-N \text { even } \\ 0 & M-N \text { odd }\end{cases}
$$


Notice that in all of the above equations the coefficients corresponding to conformal partial waves of operators with $M-N$ odd vanish which is an important check on the calculations. Such representations may not occur in the OPE of identical operators $T$.

\subsection{The large $\mathrm{N}$ AdS dual four-point function}

The four-point function calculated using supergravity on $A d S_{7} \times S^{4}$ was found in [16]. It can be written in terms of a function of two variables as:

$$
\begin{aligned}
<T T T T> & =\lambda^{-4} g_{13}^{2} g_{24}^{2} \mathcal{F}[F] \\
F\left(X_{1}, X_{2}\right) & =F_{0}\left(X_{1}, X_{2}\right)+\hat{F}\left(X_{1}, X_{2}\right) \\
\hat{F}\left(X_{1}, X_{2}\right) & =-\frac{B \lambda^{2}}{2 u v}\left(1-u \partial_{u}\right)\left(1-v \partial_{v}\right)\left(2+u \partial_{u}+v \partial_{v}\right)\left(1+u \partial_{u}+v \partial_{v}\right)\left(u v \partial_{u v}\right) \Phi \\
\Phi & =\frac{1}{\lambda}\left(\ln X_{1} X_{2} \ln \frac{1-X_{1}}{1-X_{2}}+2 \operatorname{Li}_{2}\left(X_{1}\right)-2 \operatorname{Li}_{2}\left(X_{2}\right)\right)
\end{aligned}
$$

where $F_{0}$ is the free theory function (100) and $u:=X_{1} X_{2} v:=\left(1-X_{1}\right)\left(1-X_{2}\right)$. The coefficients are given by

$$
A=1 \quad B=\frac{1}{N^{3}}
$$

so we are here considering the large $N$ expansion of the theory around the free theory with $A=1, B=0$ obtained for $N \rightarrow \infty$ with first order corrections proportional to $1 / N^{3}$. The function $\hat{F}\left(X_{1}, X_{2}\right)$ has the form

$$
\hat{F}\left(X_{1}, X_{2}\right)=F_{c}\left(X_{1}, X_{2}\right)+\log \left(\mathrm{X}_{1} \mathrm{X}_{2}\right) \mathrm{F}_{\mathrm{d}}\left(\mathrm{X}_{1}, \mathrm{X}_{2}\right)
$$

where $F_{c}, F_{d}$ contain no $\log$ terms. The log term appears from the expansion of anomalous dimensions depending on a parameter to first order in that parameter. The function $F_{d}$ therefore contains information about the anomalous dimensions of operators in the large $N$ limit whilst the function $F_{c}$ contains information about the renormalisation of the OPE coefficients.

We first perform a conformal partial wave analysis of $F_{d}$ to give the anomalous dimensions. We find that under the decomposition of $F_{d}$ into $G, f, g, A$ according to (87), only the function $G$ is non-zero.

By a similar procedure to that used in the free theory case we find the following coefficients for $M-N$ even

$$
B_{M N}=-\frac{B}{24} \frac{(M+3) !(M+4) !}{(2 M+5) !} \frac{(N+2) !(N+4) !(N-1)_{3}}{(2 N+1) !} \frac{1}{M-N+1}\left(1+\frac{(N-2)(N+1)}{2(M+N+4)(M-N+3)}\right)
$$

The anomalous dimensions are given by dividing by the free theory coefficients $A_{M N}$ (111) with $B=0$

$$
\gamma_{M N}=\frac{B_{M N}}{A_{M N}}=-\frac{3 B}{A}\left(1+\frac{(N-2)(N+1)}{2(M+N+4)(M-N+3)}\right) \frac{(N-1)_{6}}{(M-N+1)(M-N+2)(M+N+5)(M+N+6)} .
$$

Note that non-vanishing anomalous dimensions first appear for $N=2$ which correspond to the first long operators in the theory. We may read off the anomalous dimension of the operator $\mathcal{O}_{22}^{4}$ (a long operator with lowest component a scalar of dimension 8 in the free theory) $\gamma_{22}=-24 B / A$ in agreement with the results of [16]. 
Next we analyse the function $F_{c}$ to obtain information regarding the OPE coefficients. Firstly we decompose $F_{c}$ into the functions $G, f, g, A$ according to (87). We obtain

$$
g(X)=2 B X F_{21}(2,1 ; 4 ; X)-B\left(X+\frac{X}{1-X}\right) .
$$

But $F_{21}(2,1 ; 4 ; X)$ is the conformal partial wave of the energy-momentum tensor (see (97)) and

$B\left(X+\frac{X}{1-X}\right)$ is the contribution to $g$ of the free theory (see (103)) and so one finds that of all operators with charge 2, only the energy-momentum tensor contributes to the four-point function in the large $\mathrm{N}$ limit. This fact was previously observed in [16].

The analysis of $f$ gives the contribution of $\hat{F}$ to the normalisation coefficients $A_{M 0}$. These are given by

$$
\hat{A}_{M 0}=B \frac{(M+3) !^{2}(M+4)}{(2 M+5) !(M+1)} \quad M=0,2,4 \ldots
$$

and should be added to the corresponding free theory expression (109). Analysis of $G$ leads to

$$
\hat{A}_{M 1}=-3 B \frac{(M+3) !(M+4) !\left(3 M^{2}+21 M+28\right)}{M(M+2)(M+5)(2 M+5) !} \quad M=1,3,5, \ldots
$$

which should also be added to the corresponding free theory expression in order to get the full coefficient.

For $N \geq 2$ the analysis breaks down: the expressions get more and more complicated, and the coefficients no longer vanish for $M-N$ odd. This may be due to non-trivial mixing between long operators with anomalous dimensions and the phenomenon also occurs in the four dimensional $N=4$ SYM theory.

\section{Crossing symmetry}

The four point function of four energy momentum multiplets has an additional symmetry 'crossing symmetry'. This simply states that the four-point function is invariant under permutation of the insertion points. It turns out that crossing symmetry has a very simple action on the function $F\left(X_{1}, X_{2}\right)$.

Consider the four-point function (26). Acting on the variable $Z$ the permutations are generated by the two transformations $Z \rightarrow 1-Z$ and $Z \rightarrow 1 / Z$. Under $Z \rightarrow 1-Z, \mathcal{S}$ is invariant as can be seen from (77), the differential function $\Delta$ is invariant. This transformation corresponds to $X_{1} \leftrightarrow X_{3}$ and so the prefactor of the four-point function $\left(g_{13} g_{24}\right)^{2} \lambda^{-4}$ is invariant. So we have that

$$
F\left(1-X_{1}, 1-X_{2}\right)=F\left(X_{1}, X_{2}\right) .
$$

Under $Z \rightarrow Z^{-1}, \mathcal{S} \rightarrow \mathcal{S} /\left(X_{1} X_{2} Y_{1} Y_{2}\right)^{2}$, as can be easily seen from (77). The differential functional is invariant and since this transformation corresponds to $X_{1} \leftrightarrow X_{4}$ the prefactor is multiplied by $\left(Y_{1} Y_{2}\right)^{2}$. Thus we find that

$$
F\left(1 / X_{1}, 1 / X_{2}\right)=\left(X_{1} X_{2}\right)^{2} F\left(X_{1}, X_{2}\right)
$$

Note that one can easily see that the free theory function (100) satisfies these symmetries. It is harder to see but also true that the large $\mathrm{N}$ function $\hat{F}$ satisfies these symmetries. 


\section{The four point function rewritten}

In order to compare our results with others it is useful to give the four point function in the following form:

$$
\begin{aligned}
<2222>= & a_{1} \times g_{12}^{2} g_{34}^{2}+a_{2} \times g_{13}^{2} g_{24}^{2}+a_{3} \times g_{14}^{2} g_{23}^{2}+ \\
& b_{1} \times g_{13} g_{24} g_{23} g_{14}+b_{2} \times g_{12} g_{34} g_{14} g_{23}+b_{3} \times g_{12} g_{34} g_{13} g_{24} \\
= & g_{13}^{2} g_{24}^{2}\left(a_{1} \frac{Y_{1}^{2} Y_{2}^{2}}{X_{1}^{4} X_{2}^{4}}+a_{2}+a_{3} \frac{\left(1-Y_{1}\right)^{2}\left(1-Y_{2}\right)^{2}}{\left(1-X_{1}\right)^{4}\left(1-X_{2}\right)^{4}}\right. \\
& \left.\quad+b_{1} \frac{\left(1-Y_{1}\right)\left(1-Y_{2}\right)}{\left(1-X_{1}\right)^{2}\left(1-X_{2}\right)^{2}}+b_{2} \frac{Y_{1} Y_{2}\left(1-Y_{1}\right)\left(1-Y_{2}\right)}{X_{1}^{2} X_{2}^{2}\left(1-X_{1}\right)^{2}\left(1-X_{2}\right)^{2}}+b_{3} \frac{Y_{1} Y_{2}}{X_{1}^{2} X_{2}^{2}}\right)
\end{aligned}
$$

where $a_{i}, b_{i}$ are two-variable functions of $X_{1}, X_{2}$.

By writing $\mathcal{S}$ in the following form

$$
\begin{aligned}
S= & X 1 X 2(1-X 1)(1-X 2)-Y 1 Y 2(X 1+X 2)(1-X 1)(1-X 2)+ \\
& Y 1^{2} Y 2^{2}(1-X 1)(1-X 2)+(1-Y 1)(1-Y 2) X 1 X 2(X 1+X 2-2)+ \\
& Y 1 Y 2(1-Y 1)(1-Y 2)(X 1+X 2-2 X 1 X 2)+(1-Y 1)^{2}(1-Y 2)^{2} X 1 X 2
\end{aligned}
$$

in the expression for the four-point function (26) we can read off the forms of the functions $a_{i}, b_{i}$

$$
\begin{aligned}
\lambda^{4} a_{1} & =u^{4}\left(\boldsymbol{\Delta}\left(v F\left(X_{1}, X_{2}\right)\right)+\left(1-X_{1}\right)^{2} F\left(X_{1}, X_{1}\right)+\left(1-X_{2}\right)^{2} F\left(X_{2}, X_{2}\right)\right) \\
\lambda^{4} a_{2} & =\boldsymbol{\Delta}\left(u v F\left(X_{1}, X_{2}\right)\right)+X_{1}^{2}\left(1-X_{1}\right)^{2} F\left(X_{1}, X_{1}\right)+X_{2}^{2}\left(1-X_{2}\right)^{2} F\left(X_{2}, X_{2}\right) \\
\lambda^{4} a_{3} & =v^{4}\left(\boldsymbol{\Delta}\left(u F\left(X_{1}, X_{2}\right)\right)+\left(X_{1}\right)^{2} F\left(X_{1}, X_{1}\right)+\left(X_{2}\right)^{2} F\left(X_{2}, X_{2}\right)\right) \\
\lambda^{4} b_{1} & =v^{2}\left(\boldsymbol{\Delta}\left(u\left(X_{1}+X_{2}-2\right) F\left(X_{1}, X_{2}\right)\right)-2 X_{1}^{2}\left(1-X_{1}\right) F\left(X_{1}, X_{1}\right)-2 X_{2}^{2}\left(1-X_{2}\right) F\left(X_{2}, X_{2}\right)\right) \\
\lambda^{4} b_{2} & =u^{2} v^{2}\left(\boldsymbol{\Delta}\left(\left(X_{1}+X_{2}-2 u\right) F\left(X_{1}, X_{2}\right)\right)+2 X_{1}\left(1-X_{1}\right) F\left(X_{1}, X_{1}\right)+2 X_{2}\left(1-X_{2}\right) F\left(X_{2}, X_{2}\right)\right) \\
\lambda^{4} b_{3} & =-u^{2}\left(\boldsymbol{\Delta}\left(v\left(X_{1}^{2}-X_{2}^{2}\right) F\left(X_{1}, X_{2}\right)\right)-2 X_{1}\left(1-X_{1}\right)^{2} F\left(X_{1}, X_{1}\right)-2 X_{2}\left(1-X_{2}\right)^{2} F\left(X_{2}, X_{2}\right)\right)
\end{aligned}
$$

where

$$
\lambda:=X_{1}-X_{2} \quad u:=X_{1} X_{2} \quad v:=\left(1-X_{1}\right)\left(1-X_{2}\right) .
$$

We can now compare with the results of [16] where the four-point function was found using crossing symmetry. We find that the results match except that in [16] the additional terms depending on $F\left(X_{1}, X_{1}\right)$ are absent. The method employed their involved solving differential equations for $a_{1}, a_{3}$ and $b_{2}$ coming from the superconformal Ward identities and then using crossing symmetry to obtain the other functions $a_{2}, b_{1}, b_{3}$. The equation for $a_{1}$ is solved in terms of a function $F_{A S}$ and the solution has the same form as the first term of equation (128) (with $F$ replaced by $F_{A S}$ ) but without the additional two terms. Furthermore the differential equations of [16] also demand that $F_{A S}(X, X)=0$. There is therefore a slight discrepancy between our results and those of [16] which disappears in the case that $F(X, X)=0$. It does not seem to be possible to remove this discrepancy by redefining $F$ since the kernel of $\mathcal{F}[F]$ is $F=0$ ie the four-point function is uniquely defined by $F$ (see the end of section 5.2.) Note that on the other hand it is possible to rewrite the four-point function such that the extra terms in $a_{1}$ disappear. For example if we define a function $F_{A S}$ as

$$
F_{A S}\left(X_{1}, X_{2}\right)=F\left(X_{1}, X_{2}\right)-\frac{k\left(X_{1}\right)-k\left(X_{2}\right)}{\lambda} \quad k^{\prime}(X)=F(X, X)
$$

which clearly satisfies $F_{A S}(X, X)=0$ then by noting that $\Delta\left(v k\left(X_{1}\right) / \lambda\right)=\left(1-X_{1}\right)^{2} k^{\prime}(X)$ we find that (128) becomes

$$
\lambda^{4} a_{1}=u^{4} \boldsymbol{\Delta}\left(v F_{A S}\right)
$$


The equation for $a_{1}$ has been simplified, the last two terms of (128) being absent. However the redefinition (135) does not in general remove the last two terms from all of equations (128-133). In fact it removes these additional terms from $a_{1}, a_{3}$ and $b_{2}$ but at the cost of adding additional complicated $k$ dependent terms to the other three coefficient functions. The crossing symmetry conditions $(123,124)$ for $F_{A S}$ will be more complicated than they were for $F$ and will involve the function $k(X)$ as well as $F_{A S}$ (but they will reproduce the crossing symmetry relations $(123,124)$ when $k^{\prime}(X)=0$.) In general then it seems one can only completely remove the additional terms in the case that $F(X, X)=0$. The function leading to the large $N$ four-point function $\hat{F}$ (114) does satisfy $\hat{F}(X, X)=0$ so the results of [16] concerning the large $N$ four-point function are not affected by this discussion.

\section{Discussion of protected operators in the $(2,0)$ theory}

In $N=4$ super Yang-Mills there are operators which were originally assumed to be unprotected from renormalisation, but which were discovered to have vanishing anomalous dimensions using AdS/CFT. As representations in the free theory these operators lie at the threshold of the continuous series a) (179). There are in fact two types of operator which lie at this threshold, ones like the Konishi operator which develop anomalous dimensions, and other ones, which don't. In [15] it was proved that operators which occur in the OPE of two half-BPS operators and which saturate the series a) bound are protected. The three-point function of two half-BPS operators and the operator in question was examined and it was shown that an anomalous dimension for the third operator would give a 3-point function, incompatible with the superconformal symmetry.

In [26] this phenomenon was explained by a completely different and very simple argument, making use of the classical interacting theory. Operators which are defined in terms of the chiral primary operators and lie at the threshold of the unitary bound are short supermultiplets in the classical interacting theory and can not become long through the process of quantisation ${ }^{6}$. Since superconformal representation theory tells us that the operator with an anomalous dimension must be a long supermultiplet we conclude that all operators defined in terms of half-BPS operators and which saturate the unitary bound are protected. Those which can't be defined this way (such as Konishi) are long and hence unprotected. In terms of the AdS/CFT correspondence, the protected operators correspond to multi-particle supergravity states and the unprotected operators to string states.

Note that this second classification is much more general as one is not restricted to operators obtained in the OPE of two half-BPS operators.

The obvious question arises as to whether there is a similar phenomenon in six dimensions. In this case there is no known classical interacting theory so the general arguments of [26] can not be applied. Furthermore none of the operators occurring in the OPE of two CPOs lies at the threshold of the series a) so the second method can not be straightforwardly applied either. However, one may try to generalise the argument from three-point function selection rules to analyse the three-point function of more complicated operators and find protected operators in this way. We shall illustrate this with an example below.

\footnotetext{
${ }^{6}$ There do exist apparently short operators in the classical theory which develop anomalous dimensions (for example the quarter BPS descendant of the Konishi operator) but this is achieved because they can be written as descendants of a long operator. This can not happen for operators defined in terms of the half-BPS operators.
} 
Firstly however we consider the simple case of the three-point function of two CPOs and an arbitrary third operator. This case was first considered in [15] using a different method.

We have

$$
\mathcal{T} \underline{A}<\mathcal{O}_{\underline{A}}^{Q} \mathcal{O}^{p} \mathcal{O}^{q}>\sim g_{12}^{\frac{1}{2}(Q+p-q)} g_{12}^{\frac{1}{2}(Q+q-p)} g_{12}^{\frac{1}{2}(q+p-Q)}\left(X_{123}^{-1}\right)_{\underline{A}} \mathcal{T}^{\underline{A}} .
$$

The first thing to notice is that on the right hand side the representation $\mathcal{R}$ carried by $\mathcal{T}$ must be given by the Young tableau with the quantum numbers of $\mathcal{R}$ as follows (see (182)): $n_{1}=n_{3}=0$ and $a_{1}, b$ are even. This is because it is made from the tensor product of one object with two antisymmetric indices. Note that in this section we are using Young tableau in the form of (182) rather than the alternative form of (185). We must also consider restrictions due to analyticity of the left hand side. The largest poles in the variables $y_{12}$ and $y_{23}$ and the restrictions they give are

$$
\begin{array}{lll}
\left(y_{12}^{2}\right)^{\frac{1}{2}(Q+p-q)}\left(y_{12}^{-2}\right)^{\frac{b}{2}}\left(y_{12}^{-1}\right)^{\frac{a_{1}}{2}} & \Rightarrow Q+p-q \geq a_{1}+b \\
\left(y_{13}^{2}\right)^{\frac{1}{2}(Q+q-p)}\left(y_{13}^{-2}\right)^{\frac{b}{2}}\left(y_{13}^{-1}\right)^{\frac{a_{1}}{2}} & \Rightarrow Q+q-p \geq a_{1}+b \\
\left(y_{23}^{2}\right)^{\frac{1}{2}(p+q-Q)}\left(y_{23}^{2}\right)^{\frac{(b-4)}{2}} & \Rightarrow p+q-Q+b \quad \text { if } \mathrm{b} \geq 4 \\
\left(y_{23}^{2}\right)^{\frac{1}{2}(p+q-Q)} & \Rightarrow p+q-Q \geq 0 \quad \text { if } \mathrm{b}=0,2
\end{array}
$$

These results are consistent with the results of [15]. Note that since $b$ is even we can have no representations at the threshold of the series a) bounds.

In order to obtain such operators we consider the three-point function of one half-BPS operator, $\mathcal{O}^{q}$, one other series d) operator with two antisymmetric superindices, $\mathcal{O}_{[A B]}^{p}$, and a third operator which for simplicity we choose to carry $S L(4 \mid 2)$ representation given by the Dynkin indices $\mathcal{R}=\left[000(3+2 \gamma) a_{1}\right]$ (for the related Young tableau see (182)). From the general three-point function formula (12) we find

$$
\begin{aligned}
& \mathcal{T}^{\underline{B}} \mathcal{T}^{A_{1} A_{2}}<\mathcal{O}_{\underline{B}}^{Q} \mathcal{O}_{A_{1} A_{2}}^{p} \mathcal{O}^{q}>\sim g_{12}^{\frac{1}{2}(Q+p-q)} g_{13}^{\frac{1}{2}(Q+q-p)} g_{23}^{\frac{1}{2}(q+p-Q)} \times \\
& \left(X_{12}^{-1}\right)_{A_{1} C_{1}}\left(X_{12}^{-1}\right)_{A_{2} C_{2}} t\left(X_{123}\right)_{\underline{B}}^{C_{1} C_{2}} \mathcal{T} \underline{C} \mathcal{T}^{A_{1} A_{2}} \text {. } \\
& t\left(X_{123}\right)_{\underline{B}}^{C_{1} C_{2}}=a\left(X_{123}^{-1}\right)_{\underline{B}}^{n}\left(X_{123}\right)^{A_{1} A_{2}}+b\left(X_{123}^{-1}\right)_{\underline{B}_{3}}^{n} \delta_{B_{1}}^{A_{1}} \delta_{B_{2}}^{A_{2}}
\end{aligned}
$$

where $n=4 \gamma+6+a_{1}$ is the number of boxes in the Young tableau for $\mathcal{R}$ and $a, b$ are arbitrary coefficients. The indices $C_{3}$ are forced into the subrepresentation $\mathcal{R}^{\prime}=\left[000(2+2 \gamma) a_{1}\right]$. Again $a_{1}$ is forced to be even.

The lower bound on $Q$ comes from examining the pole structure in $y_{23}$. There are potential poles in the propogator term that can be potentially cancelled by zeros in $X_{123}^{-1}=-X_{13}^{-1} X_{32} X_{21}^{-1}$. When $a=0$ we find the highest pole in $y_{23}$ is

$$
\begin{array}{llll}
\left(y_{23}^{2}\right)^{\frac{1}{2}(p+q-Q)}\left(y_{23}^{2}\right)^{\gamma-1} & \Rightarrow & Q-2 \gamma \leq p+q-2 & \gamma \geq 1 \\
\left(y_{23}^{2}\right)^{\frac{1}{2}(p+q-Q)} & \Rightarrow & Q \leq p+q & \gamma=0
\end{array}
$$

When $b=0$ on the other hand we have an extra pole in $y_{23}$ from the term $X_{123}$. Therefore for this term to be analytic the charge $Q$ is even more restricted and we require $Q \leq p+q-2$ even for $\gamma=0$. Now the number $Q-2 \gamma$ is independent of the anomalous dimension $\gamma$ and so an operator with $Q=p+q, \gamma=0$ can not develop an anomalous dimension.

Having shown that certain operators on the unitary bound are protected we now discuss how other operators also saturating the bound might develop anomalous dimensions. In free $N=4$ SYM, an operator saturating the unitary bounds is short and so in order for it to develop an 
anomalous dimension it must become long by combining with other operators $[28,48]$. The same is true in the present case and analytic superspace provides a simple way of seeing which operators can combine in this way. One simply needs to look at the corresponding isotropy groups and which representations can combine to form long representations. A nice way to find this is by considering the limit as one lets $b->3$ in the representation of the isotropy group for the long field in question. One gets different answers depending on which representation for the Young tableau you take (see section B.4). These two answers give the two short operators which make up the long operator.

The simplest example is the superfield with Dynkin labels [000b00] which has lowest component a scalar $U S p(4)$ singlet operator of dimension $2 b$. As we let $b->3$, using the standard Young tableau of (182) and letting $b \rightarrow 3$ we arrive at a tableau with 2 columns and three rows corresponding to the operator lying on the threshold of the unitary bounds. Using the alternative description of the Young tableau however (185) we get a tableau with one column and four rows. This corresponds to an operator with Dynkin labels [000040] which has lowest component a scalar in the $U s p(4) 35$ representation and has dimension 8. It lies in the series d) series in the free theory but will not be protected from renormalisation.

More generally we have that the limit as $b \rightarrow 3$ of the representation with Dynkin labels $\left[n_{1} n_{2} n_{3} x a_{1} a_{2}\right]$ (where $x=b+n_{1}+n_{2}+n_{3} \rightarrow 3+n_{1}+n_{2}+n_{3}$ ) will split into two short representations as follows:

$$
\begin{array}{rlll}
{\left[n_{1} n_{2} n_{3} x a_{1} a_{2}\right]} & \oplus\left[\left(n_{1}-1\right) n_{2} n_{3}(x-1)\left(a_{1}+1\right) a_{2}\right] n_{1} \geq 1 \\
{\left[0 n_{2} n_{3} x a_{1} a_{2}\right]} & \oplus\left[0\left(n_{2}-1\right) n_{3}(x-2) a_{1}+1 a_{2}\right] & n_{1}=0 n_{2} \geq 1 \\
{\left[00 n_{3} x a_{1} a_{2}\right]} & \oplus & {\left[00\left(n_{3}-1\right) n_{3}\left(a_{1}+1\right) a_{2}\right]} & n_{1}=n_{2}=0 n_{3} \geq 1 \\
{\left[0003 a_{1} a_{2}\right]} & \oplus & {\left[0000\left(a_{1}+4\right) a_{2}\right]} & n_{1}=n_{2}=n_{3}=0 .
\end{array}
$$

Note that in SYM one has both primed and unprimed indices and therefore in general one has four short operators combining to form a long operator (for example the Konishi operator.) This is because we have two operators for each index type. Here there is only one index type and hence only two operators combine to form a long operator.

\section{Conclusion}

We have introduced the study of superconformal theories in six dimensions using the analytic superspace formalism. Analytic superspace is particularly suited to this task as it keeps all the superconformal symmetry manifest from the beginning and has a similar structure to ordinary Minkowski space so techniques can be readily adapted from that context. In particular we have shown how to find all correlation functions in the theory on analytic superspace.

We examined in detail the four-point function of four energy-momentum multiplets. We found that there were two different ways of expanding the four-point function both of which were useful. The Schur polynomial expansion was useful in order to find a nice form for the fourpoint function in terms of a single function of two invariants. Another basis, $T_{\mathcal{R}}$, which is a supersymmetric generalisation of the Jack polynomials introduced in [44], was found to be useful in order to find the partial wave expansion. We then performed a complete conformal partial wave analysis of the free theory and the supergravity dual theory, in particular giving the anomalous dimensions of all operators in the supergravity dual theory. 
We confirm that, as pointed out in [16], the free theory appears to be disconnected from the supergravity dual theory since all operators with charge 2 (other than the energy-momentum multiplet $T$ ) occurring in the OPE of two Ts have disappeared from the spectrum. This also happens in $N=4 \mathrm{SYM}$ where the operators are conjectured to acquire infinite anomalous dimensions in this limit, but here the operators are protected by the superconformal unitary bounds (and it does not seem to be possible to combine them with other operators to form long operators as happens for example in the case of certain quarter BPS operators in $N=4$ SYM $[49,50]$.

In [16] it was also noted that the function $F$ describing the four-point functions in the supergravity dual theory can be written in the form $F=F_{0}+D F_{S Y M}$ where $D$ is a differential operator and $F_{S Y M}$ is the corresponding function describing the four-point function of energy-momentum multiplets in $N=4 \mathrm{SYM}$ at large N, and large 't Hooft coupling $\lambda$. Since in the four-dimensional theory there should be a smooth deformation connecting the free theory and the large $N$ theory provided by $F_{S Y M}(\lambda)$ where $F_{S Y M}(0)=0$ and $F_{S Y M}(\infty)=F_{S Y M}$, the interesting suggestion was made that one consider the object

$$
F=F_{0}+D F_{S Y M}(\lambda)
$$

which should provide a similar deformation for the six-dimensional theory. It turns out however that on analysing the one loop four-point function derived from this deformation using the CPWA one finds that the (protected) charge 2 operators attain anomalous dimensions in conflict with unitary bounds. The resolution of the apparent conflict between the existence of this deformation and the disappearance of the charge 2 operators from the spectrum is that the deformation (146) does not respect unitarity for all values of the coupling.

Finally we considered operators at the threshold of the unitary bound a) and found examples of operators which must be protected. Notice that the protected operators in question have their $Q$ charge (183) equal to the sum of the $Q$-charges of the other two operators in the three point function. This suggests that this rule can be generalised. One conjectures for example that any operator with charge $Q$ which lies in the OPE of two protected operators of charges $p$ and $q$ where $Q=p+q$ and which saturates the series a) unitary bounds is itself protected.

\section{Acknowledgements}

We would like to thank P. Howe for many helpful discussions.

\section{A Properties of $\gamma$ matrices in six dimensions}

In six space-time dimensions one has $8 \times 8$ gamma matrices, $\Gamma^{a}$ satisfying the Clifford algebra $\Gamma^{a} \Gamma^{b}+\Gamma^{b} \Gamma^{a}=2 \eta^{a b}$ where we choose a mostly minus space-time signature. The gamma matrices can be chosen such that

$$
\Gamma^{a}=\left(\begin{array}{cc}
0 & \left(\gamma^{a}\right)_{\alpha \beta} \\
\left(\gamma^{a}\right)^{\alpha \beta} & 0
\end{array}\right)
$$

where $\left.\left(\gamma^{a}\right)_{\alpha \beta}=\frac{1}{2} \epsilon_{\alpha \beta \gamma \delta}(\gamma)^{a}\right)^{\gamma \delta}$ and so the Clifford algebra becomes

$$
\left(\gamma^{a}\right)_{\alpha \beta}\left(\gamma^{b}\right)^{\beta \gamma}+\left(\gamma^{b}\right)_{\alpha \beta}\left(\gamma^{a}\right)^{\beta \gamma}=2 \delta_{\alpha}^{\gamma} \eta^{a b}
$$


which implies that

$$
\begin{aligned}
\epsilon_{\alpha \beta \gamma \delta} x^{\alpha \beta} x^{\gamma \epsilon} & =-2 x^{2} \delta_{\delta}^{\epsilon} \\
\epsilon_{\alpha \beta \gamma \delta} x^{\alpha \beta} x^{\gamma \delta} & =-8 x^{2} .
\end{aligned}
$$

Another useful formula is

$$
\operatorname{det}\left(x^{\alpha \beta}\right)=\left(x^{2}\right)^{2}
$$

\section{B Analytic superspace in $6 \mathrm{~d}$}

\section{B.1 Supercoset spaces of the superconformal group}

In [18] (see also [20]) it was shown that all complexified superspaces of interest for studying globally supersymmetric theories in four-dimensions can be viewed as supercoset spaces of the complexified superconformal group. Then in $[22,23]$ it was observed that these could all be represented by putting crosses on a super Dynkin diagram from which one could also read off the transformation properties of superfields.

We follow the same route now to discuss six-dimensional superspaces. The complexified sixdimensional $(N, 0)$ superconformal group is $O s p(8 \mid 2 N)$ which has bosonic subgroup $S O(8) \times$ $S p(2 N)$. The corresponding Lie algebra, $\mathfrak{o s p}(8 \mid 2 N)$ can be represented as the set of $(8 \mid 2 N) \times$ $(8 \mid 2 N)$ matrices satisfying

$$
\mathfrak{o} \mathfrak{s p}(8 \mid 2 N)=\left\{M \mid M J+J M^{S T}=0\right\}
$$

where $M^{S T}$ denotes the supertanspose of $M$ and where

$$
J=\left(\begin{array}{cc|cc}
0_{4} & 1_{4} & 0 & 0 \\
1_{4} & 0_{4} & 0 & 0 \\
\hline 0 & 0 & 0_{N} & -1_{N} \\
0 & 0 & 1_{N} & 0_{N}
\end{array}\right)
$$

A general element of $\mathfrak{o s p}(8 \mid 2 N)$ therefore has the form

$$
\left(\begin{array}{cc|cc}
A & B & \alpha & \beta \\
C & -A^{T} & \Gamma & \Delta \\
\hline-\Delta^{T} & -\beta^{T} & E & F \\
\Gamma^{T} & \alpha^{T} & G & -E^{T}
\end{array}\right) \quad B+B^{T}=C+C^{T}=F-F^{T}=G-G^{T}=0
$$

Complexified super Minkowski space is an open subset of the coset space $P \backslash O s p(8 \mid 2 N)$ where $P$ is the parabolic subgroup given by matrices of the form

$$
P=\left\{\left(\begin{array}{cc|c}
\bullet & 0 & 0 \\
\bullet & \bullet & \bullet \\
\hline \bullet & 0 & \bullet
\end{array}\right)\right\}
$$

where bullets denote non-zero elements. Minkowski superspace has coordinates $\left(x^{\alpha \beta}, \theta^{\alpha i}\right)$ where $\alpha, \beta=1, \ldots 4 ; i=1 \ldots 2 N$ and $x^{\alpha \beta}=-x^{\beta \alpha}$. A coset representative is given by 


$$
s(x, \theta)=\left(\begin{array}{cc|cc}
1 & x^{\alpha \beta} & \theta^{\alpha s} & \theta^{\alpha s^{\prime}} \\
0 & -1 & 0 & 0 \\
\hline 0 & \theta_{r}{ }^{\beta} & 1 & 0 \\
0 & \theta_{r^{\prime}}{ }^{\beta} & 0 & -1
\end{array}\right)
$$

where $\theta_{i}^{\alpha}=\eta_{i j} \theta^{\alpha j}$ and where we have split the indices $i=\left(r, r^{\prime}\right)$ with $r \in\{1,2\}, r^{\prime} \in\{3,4\}$.

The super Dynkin diagram for $O s p(8 \mid 2 N)$ in the form compatible with super Minkowski space is given $\mathrm{by}^{7}$

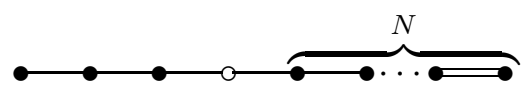

Then all superspaces of interest can be represented by putting crosses on the nodes of this diagram. For instance, super Minkowski space is specified by putting a single cross through the odd node (as in four dimensions).

In order to more easily see what the parabolic subgroups look like it is convenient to change the basis to a more convenient form as follows

$$
\left(\begin{array}{c}
v^{\alpha} \\
v_{\alpha} \\
\hline v^{a} \\
v_{a}
\end{array}\right) \rightarrow\left(\begin{array}{c}
\frac{v^{\alpha}}{v^{a}} \\
v_{\tilde{a}} \\
v_{\alpha}
\end{array}\right)
$$

where $v_{\tilde{k}}=v_{(N-k)}$. In this basis the parabolic subalgebras are simply given by block lower triangular matrices. For instance, for super Minkowski space we have

$$
P=\left\{\left(\begin{array}{c|c|c}
\bullet & & \\
\hline \bullet & \bullet_{2 N} & \\
\hline \bullet & \bullet & \bullet
\end{array}\right)\right\}
$$

where the subscripts indicate square matrices with the given size.

More generally, the Levi subgroup specified by putting crosses through the nodes $\left(k_{1}, k_{2}, \ldots k_{p-1}\right)$, with $\left(k_{p-1}<N+4\right)$, of the Dynkin diagram is

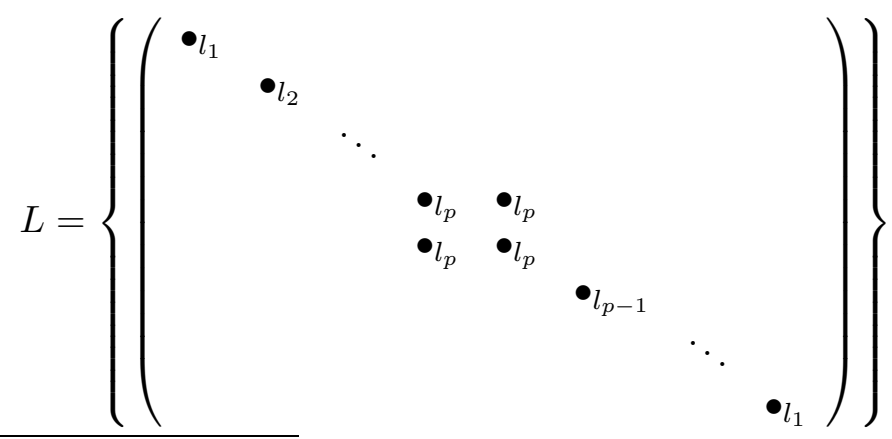

\footnotetext{
${ }^{7}$ Unlike for the purely bosonic case, there can be different super Dynkin diagrams for the same supergroup. Different Dynkin diagrams lead to do different choices of simple roots, and hence to different possible parabolic subgroups. The Dynkin diagram here leads to the parabolic subgroup $P$ for super Minkowski space.
} 
where $l_{i}=k_{i}-k_{i-1}$ and we define $k_{0}=0, k_{p}=N+4$. The corresponding parabolic subgroup is just the union of $L$ with the set of lower triangular matrices. If there is a cross through the final node also, so the nodes $\left(k_{1}, k_{2}, \ldots k_{p-1}, N+4\right)$ are crossed through, then the Levi subgroup has the form

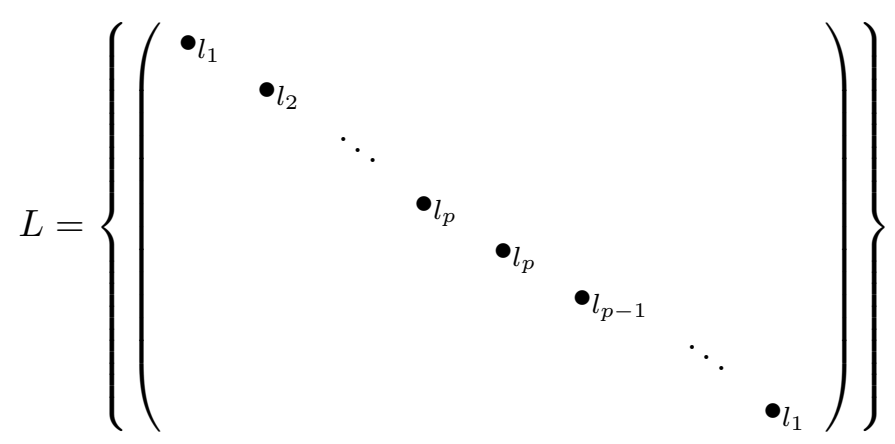

and again, the parabolic subgroup consists of the union of this set of matrices, with the lower triangular matrices.

\section{B.2 Harmonic and analytic superspaces}

We will be interested in Harmonic superspace and their related analytic superspaces. Harmonic superspaces have super Dynkin diagrams with a cross through the odd node and any number of further crosses through the nodes to the right of the odd node. The related analytic superspaces have the same Dynkin diagram, but with the odd node no longer crossed through.

In particular we can define $(N, p)$ harmonic superspaces by the Dynkin diagram with just two crossed through nodes, the odd node and the $p$ th internal node.

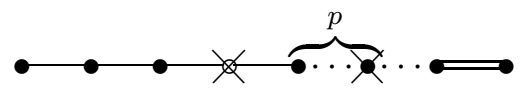

To find the coordinates of these spaces we split the internal index as follows $a=\left(r, r^{\prime}\right), r=$ $1 \ldots p, r^{\prime}=p+1, \ldots N$, then the coordinates of $(N, p)$ harmonic superspace are

$$
\left(x^{\alpha \beta}, \theta_{b}^{\alpha}, \theta^{\alpha b}, y_{s^{\prime}}^{r}, y^{r s^{\prime}}, y^{r s}\right)
$$

and one can see that this space has the form Minkowski space times an internal manifold.

The related analytic superspace has the Dynkin diagram

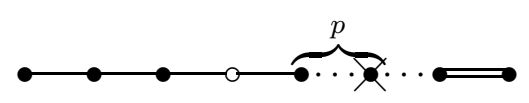

This has coordinates

$$
\left(x^{\alpha \beta}, \theta_{s^{\prime}}^{\alpha}, \theta^{\alpha a}, y_{s^{\prime}}^{r}, y^{r s^{\prime}}, y^{r s}\right)
$$

and we see that this has the form of Harmonic superspace but with fewer odd coordinates. In fact there are only $4(2 N-p)$ odd coordinates instead of the maximal $8 N$. 
More general $(N, p)$ harmonic superspaces can also be defined which have the same two crosses through the odd node and the $p$ th internal node, but with further crosses through nodes to the right of the $p$ th internal node. The corresponding analytic superspaces will still have $4(2 N-p)$ odd coordinates, but will have a different internal space.

Of most interest for us will be $(N, N)$ analytic superspace which just has one cross through the final $N$ th node

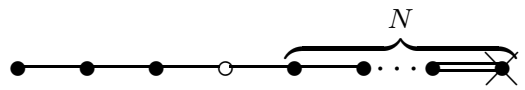

This has coordinates $\left(x^{\alpha \beta}, \lambda^{\alpha b}, y^{a b}\right)$ and we see that there are only $4 N$ odd coordinates.

If we define the superindex $A=(\alpha, a)$, then the parabolic subalgebra for $(N, N)$ harmonic superspace is given by

$$
\mathfrak{p}=\left\{\left(\begin{array}{cc}
-A^{A} & 0 \\
-C_{A B} & D_{A}{ }^{B}
\end{array}\right)\right\}
$$

where

$$
D_{A}^{B}=(-1)^{A(A+B)} A_{A}^{B}
$$

ie

$$
D=A^{S T}
$$

and all three entries are $(4 \mid N) \times(4 \mid N)$ matrices. As in the four dimensional case we choose $\alpha$ to be even and $a$ to be odd.

A coset representative for the space is

$$
s(X)=\left(\begin{array}{cc}
1 & X \\
0 & -1
\end{array}\right)
$$

where the components of $X$ are

$$
X=\left(\begin{array}{c|c}
x^{\alpha \beta} & \lambda^{\alpha b} \\
\hline-\lambda^{a \beta} & y^{a b}
\end{array}\right)
$$

Now $x^{\alpha \beta}+x^{\beta \alpha}=0, y^{a b}-y^{b a}=0$ and so we see that

$$
X^{A B}=-(-1)^{A B} X^{B A},
$$

ie. $X$ is (generalised) antisymmetric.

Using standard coset space techniques one can then show that a general infinitesimal conformal transformation

$$
\left(\begin{array}{cc}
-A & B \\
-C & A^{s T}
\end{array}\right)
$$


acting on the group leads to the following infinitesimal transformation of the coordinates $\mathrm{X}$

$$
\delta X=B+A X+X A^{s T}+X C X .
$$

Here $A, B, C, X$ are all $(4 \mid 2) \times(4 \mid 2)$ supermatrices, and $B$ and $C$ are generalised anti-symmetric.

\section{B.3 Representations of the superconformal group}

Unitary representations of the real superconformal group in six dimensions have were classified in [45].

They are given in terms of the labels,

$$
\left[d ; n_{1}, n_{2}, n_{3} ; a_{1}, \ldots, a_{N}\right]
$$

where $d$ is the conformal weight, $n_{1}, n_{2}, n_{3}$ are the Dynkin labels specifying the representation

of the six-dimensional Lorentz group $S O(5,1)$ and $a_{1}, \ldots a_{N}$ are Dynkin labels for the representation of the internal group $U s p(2 N)$. These are related to the super Dynkin coefficients of the super conformal group as follows

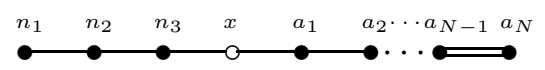

where

$$
x=\frac{d}{2}+\frac{1}{4}\left(n_{1}+2 n_{2}+3 n_{3}\right)-m_{1}
$$

where $m_{1}=\sum_{i} a_{i}$.

The unitary representations fall into four series as follows

$$
\begin{array}{ll}
\text { a) } & d \geq \frac{1}{2}\left(3 n_{1}+2 n_{2}+n_{3}\right)+2 m_{1}+6 \\
\text { b) } & d=\frac{1}{2}\left(n_{3}+2 n_{2}\right)+2 m_{1}+4, \quad n_{1}=0 \\
\text { c) } & d=\frac{1}{2} n_{3}+2 m_{1}+2, \quad n_{1}=n_{2}=0 \\
\text { d) } & d=2 m_{1}, \quad n_{1}=n_{2}=n_{3}=0 .
\end{array}
$$

In terms of super Dynkin labels these bounds become

$$
\begin{array}{ll}
\text { a) } & x \geq n_{1}+n_{2}+n_{3}+3 \\
\text { b) } & x=n_{2}+n_{3}+2, \quad n_{1}=0 \\
\text { c) } & x=n_{3}+1, \quad n_{1}=n_{2}=0 \\
\text { d) } & x=n_{1}=n_{2}=n_{3}=0 .
\end{array}
$$

Notice that for the series $d$ ) representations the first four super Dynkin labels vanish.

All series d) representations can be given as ordinary superfields (ie without superindices, although they may have internal indices) on $(N, 1)$ analytic superspace and therefore only depend on at most $4(2 N-1)$ odd coordinates. Series d) representations with $a_{1}=\ldots=a_{N-1}=0$ can be given as scalar fields in $(N, N)$ analytic superspace and hence only depend on half the total number of odd coordinates. These are the representations which are dual to Kaluza Klein states in the AdS/CFT correspondence. 


\section{B.4 Representations on analytic superspace: superindices}

We wish to describe all unitary representations as superfields (possibly with superindices) on $(N, N)$ analytic superspace. Superfields on $(N, N)$ analytic superspace carry linear representations of $\mathfrak{s l}(4 \mid N) \oplus \mathbb{C}=\mathfrak{g l}(4 \mid N)$. This linear representation can be read off from the super Dynkin diagram, and for $x \in \mathbb{Z}$ it can be obtained by tensoring together the anti-fundamental representation (all unitary representations have downstairs superindices). Tensor representations can be defined using Young tableaux.

Abstractly, a representation carried by a superfield on $(N, N)$ analytic superspace is given by the Dynkin diagram

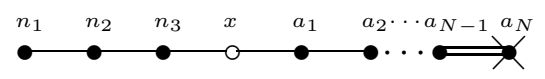

We read off the linear representation of the isotropy group $\mathfrak{s l}(4 \mid N)$ which the superfield carries. This is given by the Dynkin diagram

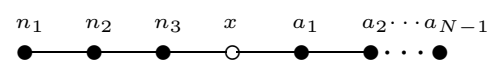

This is related to the following Young tableau

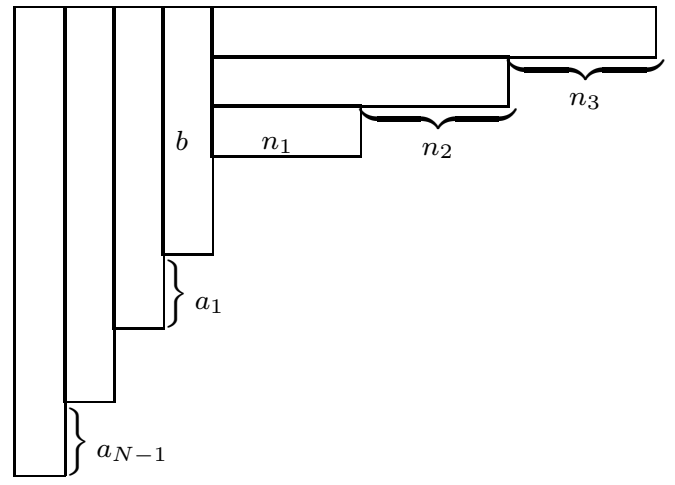

where $b=x-\left(n_{1}+n_{2}+n_{3}\right)$.

The three series of unitary bounds now come simply from demanding that the Young tableaux have the correct shape. Clearly in the generic case we require that $b \geq 3$, but if $n_{1}=0$ we are allowed $b=2$, if $n_{1}=n_{2}=0$ we can have $b=1$ and if $n_{1}=n_{2}=n_{3}=0$ we can have $b=0$, giving precisely the four series of unitary bounds above.

It will also be useful to define the quantum number $Q$

$$
Q=\left(x+m_{1}-n_{1}-n_{2}-n_{3}\right)=\left(b+m_{1}\right)
$$

which in terms of the dilation weight $d$ is

$$
Q=\frac{1}{2} d-\frac{1}{4}\left(3 n_{1}+2 n_{2}+n_{3}\right)
$$

This is sometimes referred to as 'twist' in the literature. 
Note that if $b>0$ there is a Young tableau related to (182) which corresponds to the same $\mathfrak{s l}(4 \mid N)$ representation but different $\mathfrak{g l}(4 \mid N)$ representations. This has the form

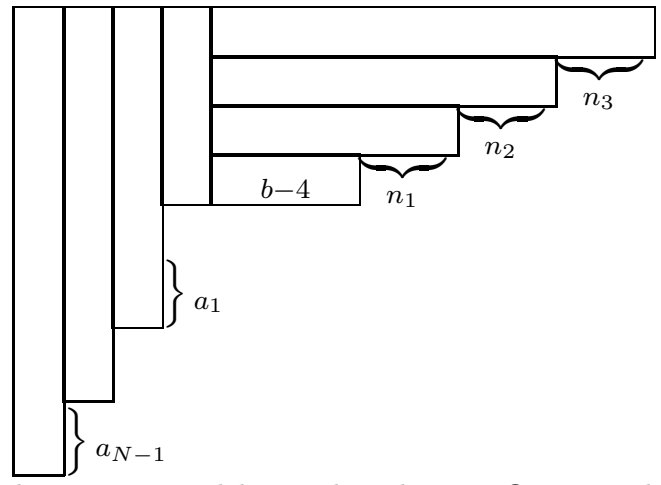

With this form of the Young tableau the charge Q is modified to

$$
Q=4+m_{1} .
$$

We use the first form of the Young tableau when discussing operators in section 9 whereas for the discussion of the four-point function we use this second form.

\section{Superconformal invariants}

The procedure for finding arbitrary four-point invariants in $N=4 \mathrm{SYM}$ given in [28] may be readily adapted to the present case. We sketch this here.

Using similar arguments to those given for the case of the four-point function around (19) one can reduce the problem of finding an n-point function $F\left(X_{1}, X_{2}, \ldots X_{n}\right)$ invariant under superconformal transformations (acting on the $X \mathrm{~s}$ as in (3)) to that of finding a function $F\left(Z_{1}, Z_{2}, \ldots Z_{n-3}\right)$ invariant under the adjoint representation of $O S p(4 \mid N)$.

One can then proceed in one of two ways. We can form an invariant function as a polynomial in the $\left(Z_{i}\right)^{A}{ }_{B}$ s and either take the superdeterminant of this or suitably contract all the indices with $\delta_{B}^{A}$ s. ${ }^{8}$ For example for the four-point function we have discussed expansions of $\mathrm{Z}$ in terms of Schur polynomials and the polynomials $T \mathcal{R}(Z)$ which are all polynomials in traces of powers of $Z$.

Another approach consists of systematically using up all remaining symmetries and thereby reducing the number of components in the $Z \mathrm{~s}$. The remaining components will be invariants ${ }^{9}$.

\footnotetext{
${ }^{8}$ Note that we may use any numerically invariant tensors we have at our disposal to construct invariants. In the present context we also have an invariant tensor $K^{A B}$ used in the definition of $O S p(4 \mid N)(22)$. We can not straightforwardly use $K$ however to contract indices since it has two upstairs indices and any object constructed from $Z \mathrm{~s}$ has an equal number of upstairs and downstairs indices. It may well, however, be possible to construct invariants by using $K \mathrm{~s}$ and $\delta \mathrm{s}$ in conjunction with an invariant $\mathcal{E}$ tensor. Such an $\mathcal{E}$ tensor was introduced in [28] in the context invariants in $N=4 \mathrm{SYM}$ and reflects the equivalence of different tensors discussed in section B.4. In the present context (for $N \neq 4$ ) such a tensor would have different numbers of upstairs and downstairs indices (unlike for $N=4 \mathrm{SYM}$ ) and so together with $K$ could lead to invariants. It would be interesting to investigate this further.

${ }^{9}$ Strictly they are only invariant under transformations connected to the identity. There may still be global discrete transformations acting on these variables. For example in the $n=4$ case we have seen that four-point functions are invariant under $X_{1} \leftrightarrow X_{2}$
} 
The first stage of this procedure consists of diagonalising $Z_{1}$ to the form of (25). In the case $n=4$ we stop here but for higher point functions we can go further. The residual infinitesimal symmetry leaving $Z_{1}$ invariant has the form:

$$
A=\left(\begin{array}{c|c}
\mathbf{a} & 0 \\
\hline 0 & 0
\end{array}\right) \quad \mathbf{a}=\left(\begin{array}{cc}
D_{1} & D_{2} \\
D_{3} & -D_{1}
\end{array}\right)
$$

where the $D_{i}$ are $2 \times 2$ diagonal matrices. One then uses up as much of this symmetry to obtain a specific form for $Z_{2}$ which may still be invariant under a smaller residual symmetry in which

case one uses this on $Z_{3}$ etc. When all the infinitesimal symmetry has been absorbed we are necessarily left with invariants (up to discrete symmetries.)

\section{References}

[1] J. Maldacena, "The large N limit of superconformal field theories and supergravity," Adv. Theor. Math. Phys. 2 (1998) 231-252, hep-th/9711200.

[2] E. Witten, "Anti-de Sitter space and holography," Adv. Theor. Math. Phys. 2 (1998) 253-291, hep-th/9802150.

[3] S. S. Gubser, I. R. Klebanov, and A. M. Polyakov, "Gauge theory correlators from non-critical string theory," Phys. Lett. B428 (1998) 105, hep-th/9802109.

[4] O. Aharony, S. S. Gubser, J. Maldacena, H. Ooguri, and Y. Oz, "Large N field theories, string theory and gravity," Phys. Rept. 323 (2000) 183, hep-th/9905111.

[5] E. D'Hoker and D. Z. Freedman, "Supersymmetric gauge theories and the AdS/CFT correspondence," hep-th/0201253.

[6] P. S. Howe and P. C. West, "AdS/SCFT in superspace," hep-th/0105218.

[7] M. F. Sohnius and P. C. West, "Conformal invariance in N=4 supersymmetric Yang-Mills theory," Phys. Lett. B100 (1981) 245.

[8] L. Brink, O. Lindgren, and B. E. W. Nilsson, "N=4 Yang-Mills theory on the light cone," Nucl. Phys. B212 (1983) 401.

[9] P. S. Howe, K. S. Stelle, and P. K. Townsend, "Miraculous ultraviolet cancellations in supersymmetry made manifest," Nucl. Phys. B236 (1984) 125.

[10] P. S. Howe, G. Sierra, and P. K. Townsend, "Supersymmetry in six-dimensions," Nucl. Phys. B221 (1983) 331.

[11] P. S. Howe, "On harmonic superspace," hep-th/9812133.

[12] P. S. Howe, "Aspects of the D = 6, (2,0) tensor multiplet," Phys. Lett. B503 (2001) 197-204, hep-th/0008048.

[13] J.-H. Park, "Superconformal symmetry in six-dimensions and its reduction to four," Nucl. Phys. B539 (1999) 599-642, hep-th/9807186. 
[14] R. Corrado, B. Florea, and R. McNees, "Correlation functions of operators and Wilson surfaces in the $\mathrm{d}=6,(0,2)$ theory in the large N limit," Phys. Rev. D60 (1999) 085011, hep-th/9902153.

[15] B. Eden, S. Ferrara, and E. Sokatchev, "(2,0) superconformal OPEs in D =6, selection rules and non-renormalization theorems," hep-th/0107084.

[16] G. Arutyunov and E. Sokatchev, "Implications of superconformal symmetry for interacting $(2,0)$ tensor multiplets," Nucl. Phys. B635 (2002) 3-32, hep-th/0201145.

[17] A. Galperin, E. Ivanov, S. Kalitsyn, V. Ogievetsky, and E. Sokatchev, "Unconstrained $\mathrm{N}=2$ matter, Yang-Mills and supergravity theories in harmonic superspace," Class.

Quant. Grav. 1 (1984) 469.

[18] G. G. Hartwell and P. S. Howe, "(N, p, q) harmonic superspace," Int. J. Mod. Phys. A10 (1995) 3901-3920, hep-th/9412147.

[19] P. S. Howe and G. G. Hartwell, "A Superspace survey," Class. Quant. Grav. 12 (1995) 1823 .

[20] J. Lukierski and A. Nowicki, "General superspaces from supertwistors," Phys. Lett. B211 (1988) 276-280.

[21] A. Galperin, E. Ivanov, V. Ogievetsky, and E. Sokatchev, "Conformal invariance in harmonic superspace,". JINR-E2-85-363.

[22] P. Heslop and P. S. Howe, "On harmonic superspaces and superconformal fields in four dimensions," Class. Quant. Grav. 17 (2000) 3743-3768, hep-th/0005135.

[23] P. J. Heslop, "Superfield representations of superconformal groups," Class. Quant. Grav. 19 (2002) 303-346, hep-th/0108235.

[24] H. Osborn and A. C. Petkou, "Implications of conformal invariance in field theories for general dimensions," Ann. Phys. 231 (1994) 311-362, hep-th/9307010.

[25] H. Osborn, "Implications of conformal invariance for quantum field theories in $d>2$," hep-th/9312176.

[26] P. J. Heslop and P. S. Howe, "A note on composite operators in N = 4 SYM," Phys. Lett. B516 (2001) 367-375, hep-th/0106238.

[27] P. J. Heslop and P. S. Howe, "OPEs and 3-point correlators of protected operators in N = 4 SYM," Nucl. Phys. B626 (2002) 265-286, hep-th/0107212.

[28] P. J. Heslop and P. S. Howe, "Aspects of N = 4 SYM," hep-th/0307210.

[29] P. J. Heslop, "Superconformal field theories in analytic superspace," hep-th/0403144.

[30] E. D'Hoker, S. D. Mathur, A. Matusis, and L. Rastelli, "The operator product expansion of N = 4 SYM and the 4-point functions of supergravity," Nucl. Phys. B589 (2000) 38-74, hep-th/9911222.

[31] B. U. Eden, P. S. Howe, A. Pickering, E. Sokatchev, and P. C. West, "Four-point functions in N = 2 superconformal field theories," Nucl. Phys. B581 (2000) 523, hep-th/0001138. 
[32] B. Eden, A. C. Petkou, C. Schubert, and E. Sokatchev, "Partial non-renormalisation of the stress-tensor four-point function in N = 4 SYM and AdS/CFT," hep-th/0009106.

[33] G. Arutyunov, S. Frolov, and A. C. Petkou, "Operator product expansion of the lowest weight CPOs in N = 4 SYM(4) at strong coupling," Nucl. Phys. B586 (2000) 547-588, hep-th/0005182.

[34] G. Arutyunov, S. Frolov, and A. Petkou, "Perturbative and instanton corrections to the OPE of CPOs in N = 4 SYM(4)," Nucl. Phys. B602 (2001) 238-260, hep-th/0010137.

[35] B. Eden, C. Schubert, and E. Sokatchev, "Three-loop four-point correlator in $\mathrm{N}=4$ SYM," Phys. Lett. B482 (2000) 309, hep-th/0003096.

[36] B. Eden, C. Schubert, and E. Sokatchev, "Four-point functions of chiral primary operators in N = 4 SYM," hep-th/0010005.

[37] M. Bianchi, S. Kovacs, G. Rossi, and Y. S. Stanev, "Anomalous dimensions in N = 4 SYM theory at order $g^{4}, "$ Nucl. Phys. B584 (2000) 216, hep-th/0003203.

[38] G. Arutyunov, B. Eden, A. C. Petkou, and E. Sokatchev, "Exceptional non-renormalization properties and OPE analysis of chiral four-point functions in $\mathrm{N}=4$ SYM(4)," hep-th/0103230.

[39] F. A. Dolan and H. Osborn, "Superconformal symmetry, correlation functions and the operator product expansion," Nucl. Phys. B629 (2002) 3-73, hep-th/0112251.

[40] P. J. Heslop and P. S. Howe, "Four-point functions in N = 4 SYM," JHEP 01 (2003) 043, hep-th/0211252.

[41] G. Arutyunov, F. A. Dolan, H. Osborn, and E. Sokatchev, "Correlation functions and massive Kaluza-Klein modes in the AdS/CFT correspondence," Nucl. Phys. B665 (2003) 273-324, hep-th/0212116.

[42] G. Arutyunov, S. Penati, A. Santambrogio, and E. Sokatchev, "Four-point correlators of BPS operators in N $=4$ SYM at order $g^{4}, "$ Nucl. Phys. B670 (2003) 103-147, hep-th/0305060.

[43] F. A. Dolan and H. Osborn, "Conformal four point functions and the operator product expansion," Nucl. Phys. B599 (2001) 459-496, hep-th/0011040.

[44] F. A. Dolan and H. Osborn, "Conformal partial waves and the operator product expansion," Nucl. Phys. B678 (2004) 491-507, hep-th/0309180.

[45] V. K. Dobrev, "Positive energy unitary irreducible representations of D $=6$ conformal supersymmetry," J. Phys. A35 (2002) 7079-7100, hep-th/0201076.

[46] G. Arutyunov, B. Eden, and E. Sokatchev, "On non-renormalization and OPE in superconformal field theories," Nucl. Phys. B619 (2001) 359-372, hep-th/0105254.

[47] F. A. Dolan, L. Gallot, and E. Sokatchev, "On Four-Point Functions of Half-BPS Operators in General Dimensions," hep-th/0405180.

[48] F. A. Dolan and H. Osborn, "On short and semi-short representations for four dimensional superconformal symmetry," Ann. Phys. 307 (2003) 41-89, hep-th/0209056. 
[49] F. A. Dolan and H. Osborn, "On short and semi-short representations for four dimensional superconformal symmetry," Ann. Phys. 307 (2003) 41-89, hep-th/0209056.

[50] E. D'Hoker, P. Heslop, P. Howe, and A. V. Ryzhov, "Systematics of quarter BPS operators in N = 4 SYM," JHEP 04 (2003) 038, hep-th/0301104. 